\title{
ERNA AND FRIEDMAN'S REVERSE MATHEMATICS
}

\author{
SAM SANDERS
}

\begin{abstract}
Elementary Recursive Nonstandard Analysis, in short ERNA, is a constructive system of nonstandard analysis with a PRA consistency proof, proposed around 1995 by Patrick Suppes and Richard Sommer. Recently, the author showed the consistency of ERNA with several transfer principles and proved results of nonstandard analysis in the resulting theories (see 12 and [13]). Here, we show that Weak König's lemma (WKL) and many of its equivalent formulations over $\mathrm{RCA}_{0}$ from Reverse Mathematics (see [21] and 22]) can be 'pushed down' into the weak theory ERNA, while preserving the equivalences, but at the price of replacing equality with equality 'up to infinitesimals'. It turns out that ERNA plays the role of $\mathrm{RCA}_{0}$ and that transfer for universal formulas corresponds to WKL.
\end{abstract}

\section{INTRODUCTION}

The theory ERNA (short for Elementary Recursive Nonstandard Analysis) was introduced around 1995 by Patrick Suppes and Richard Sommer (23] and [24]), who also proved its consistency inside PRA. ERNA's predecessor, developed by Rolando Chuaqui and Patrick Suppes ([6] and [25]), has recently been reconsidered in the systems $\mathrm{NQA}^{ \pm}$of Michal Rössler and Emil Jeřábek ([17]). In [12], a $\Pi_{1}$ transfer principle was added to ERNA, and a PRA-proof was provided for the consistency of the resulting theory. This theory provides a $\Sigma_{1}$-supremum principle and the Cauchy completeness of ERNA's field, both 'up to infinitesimals'. In [13], ERNA was further extended with $\Sigma_{2}$-transfer, with a consistency proof for the extended theory. This theory is better suited for ERNA's intended purpose, which is to develop basic analysis in a finitistic setting; in particular, transfer applies to formulas containing nonstandard objects such as ERNA's exponential $\sum_{n=0}^{\omega} \frac{x^{n}}{n !}$. Below, we upgrade transfer for universal formulas in a similar way.

Reverse Mathematics is a program in Foundations of Mathematics founded around 1975 by Harvey Friedman ([8 and 9$]$ ) and developed intensely by Stephen Simpson and others; for an overview of the subject, see 21] and [22. The goal of Reverse Mathematics is to determine what (minimal) axiom system is necessary to prove a particular theorem. By now, it is well known that large portions of mathematics (especially so in analysis) can be carried out in systems far weaker than ZFC, the 'usual' background theory for mathematics. Classifying theorems according to their logical strength reveals the following striking phenomenon: 'It turns out that, in many particular cases, if a mathematical theorem is proved from appropriately weak set existence axioms, then the axioms will be logically equivalent to the theorem' (21, Preface]). This recurring phenomenon is called the 'Main theme' of Reverse Mathematics (see e.g. 20]) and a good instance of it, is the following theorem from [21, p. 36].

University of Ghent, Department of Pure Mathematics and Computer Algebra, Krijgslaan 281, B-9000 Gent (Belgium)

E-mail address: sasander@cage.ugent.be. 
1. Theorem (Reverse Mathematics for $\mathrm{WKL}_{0}$ ). Within $\mathrm{RCA}_{0}$, one can prove that Weak König's Lemma (WKL) is equivalent to each of the following mathematical statements:

(1) The Heine-Borel lemma: every covering of [0,1] by a sequence of open intervals has a finite subcovering.

(2) Every covering of a compact metric space by a sequence of open sets has a finite subcovering.

(3) Every continuous real-valued function on $[0,1]$, or on any compact metric space, is bounded.

(4) Every continuous real-valued function on $[0,1]$, or on any compact metric space, is uniformly continuous.

(5) Every continuous real-valued function on [0,1] is Riemann integrable.

(6) The maximum principle: every continuous real-valued function on $[0,1]$, or on any compact metric space, is bounded, or (equivalently) has a supremum or (equivalently) attains its maximum.

(7) The Peano existence theorem: if $f(x, y)$ is continuous in the neighbourhood of $(0,0)$, then the initial value problem $y^{\prime}=f(x, y), y(0)=0$ has a continuously differentiable solution in the neighbourhood of $(0,0)$.

(8) Gödel's completeness theorem: every at most countable consistent set of sentences in the predicate calculus has a countable model.

(9) Every countable commutative ring has a prime ideal.

(10) Every countable field (of characteristic 0) has a unique algebraic closure.

(11) Every countable formally real field is orderable.

(12) Every countable formally real field has a (unique) real closure.

(13) Brouwer's fixed point theorem: every uniformly continuous function from $[0,1]^{n}$ to $[0,1]^{n}$ has a fixed point.

(14) The separable Hahn-Banach theorem: if $f$ is a bounded linear functional on a subspace of a separable Banach space, and if $\|f\| \leq 1$, then $f$ has an extension $\tilde{f}$ to the whole space such that $\|\tilde{f}\| \leq 1$.

Below, we will establish a similar theorem for ERNA. For future reference, we list some of the arguments pointing in this direction. First, there is an interpretation of $\mathrm{WKL}_{0}$ in ERNA $+\Pi_{1}$-TRANS (see [12, Theorem 45]). Hence it is to be expected that some of the equivalent formulations of WKL have an interpretation in ERNA too. Second, in 16], Keisler introduces a nonstandard and conservative extension of $\mathrm{WKL}_{0}$, called ${ }^{*} \mathrm{WKL}_{0}$. It is defined as ${ }^{*} \Sigma \mathrm{PA}+\mathrm{STP}$, where ${ }^{*} \Sigma \mathrm{PA}$ is a weak nonstandard first-order theory and STP is the second-order principle that any set of naturals can be coded into a hyperinteger and vice versa. As part of STP plays the role of WKL, other nonstandard principles, like $\Pi_{1}$-TRANS, may have similar properties. Third, ERNA can prove results of basic analysis 'up to infinitesimals'; see e.g. 24], where the proof of ERNA's version of the above item (7) is outlined. This suggests that replacing equality with equality up to infinitesimals might translate some of the equivalences in theorem 1 into ERNA. Fourth, a version of $\Sigma_{1}$-separation is provable in ERNA $+\Pi_{1}$-TRANS (see theorem 37). The former schema is equivalent to WKL ([21, IV.4.4]). Fifth, in [21, X.4.3] Simpson suggests reconsidering the results of Reverse Mathematics for $\mathrm{WKL}_{0}$ in the weaker theory $\mathrm{WKL}_{0}^{*}$. For ERNA, which has roughly the same first-order strength, we will prove the following theorem; it contains several statements, translated from theorem 1 and [21, IV] into ERNA's language, while preserving equivalence. For the definitions, see below. 
2. Theorem (Reverse Mathematics for ERNA $+\Pi_{1}$-TRANS). The theory ERNA proves the equivalence between $\Pi_{1}$-TRANS and each of the following theorems concerning near-standard functions:

(1) Every S-continuous function on $[0,1]$, or on any interval, is bounded.

(2) Every $S$-continuous function on $[0,1]$, or on any interval, is continuous there.

(3) Every S-continuous function on [0,1], or on any interval, is Riemann integrable.

(4) Weierstrass' theorem: every S-continuous function on $[0,1]$, or on any interval, has, or attains a supremum, up to infinitesimals.

(5) The uniform Brouwer fixed point theorem: every S-continuous function $\phi$ : $[0,1] \rightarrow[0,1]$ has a fixed point up to infinitesimals of arbitrary depth.

(6) The first fundamental theorem of calculus.

(7) The Peano existence theorem for ordinary differential equations.

(8) The Cauchy completeness, up to infinitesimals, of ERNA's field.

(9) Every $S$-continuous function on $[0,1]$ has a modulus of uniform continuity.

(10) The Weierstrass approximation theorem.

A common feature of the items in the theorem is that strict equality has been replaced with $\approx$, i.e. equality up to infinitesimals. This seems the price to be paid for 'pushing down' into ERNA the theorems equivalent to WKL. For instance, item (5) guarantees that there is a number $x_{0}$ in $[0,1]$ such that $\phi\left(x_{0}\right) \approx x_{0}$, i.e. a fixed point up to infinitesimals, but in general there is no point $y_{0}$ such that $\phi\left(y_{0}\right)=y_{0}$. In this way, one could say that the Reverse Mathematics of ERNA $+\Pi_{1}$-TRANS is a 'copy up to infinitesimals' of the Reverse Mathematics of $\mathrm{WKL}_{0}$.

Below, we prove theorem 2 in ERNA and briefly explore a possible connection between the Reverse Mathematics for ERNA $+\Pi_{1}$-TRANS and the program of Constructive Reverse Mathematics. We also demonstrate that our results have implications for physics. Recently, the question has arisen whether Reverse Mathematics has implications outside mathematics and, to the best of our knowledge, we have obtained the first example.

\section{UPGRADING TRANSFER}

For the definition of ERNA and related notation, we refer to [12]. In this section, we expand the scope of ERNA's universal transfer principle, which, until now, was quite limited. Indeed, $\Pi_{1}$-transfer is limited to formulas of $L^{s t}$. Hence, a formula cannot be transferred if it contains, for instance, ERNA's cosine $\sum_{n=0}^{\omega}(-1)^{n} \frac{x^{2 n}}{(2 n) !}$ or similar objects not definable in $L^{s t}$. This is quite a limitation, especially for the development of basic analysis. In this section, we overcome this problem by widening the scope of $\Pi_{1}$-transfer so as to be applicable to objects like ERNA's cosine. For special $\Pi_{1}$-formulas, this was done in $[13, \S 3]$ with a relatively easy proof. For general $\Pi_{1}$-formulas, the proof becomes significantly more difficult.

First we label some terms which, though not part of $L^{s t}$, are 'nearly as good' as standard for the purpose of transfer. As in [12, Notation 57], the variable $\omega^{\prime}$ in $\left(\forall \omega^{\prime}\right)$ runs over the infinite hypernaturals.

3. Definition. Let the term $\tau(n, \vec{x})$ be standard, i.e. not involve $\omega$ or $\approx$. We say that $\tau(\omega, \vec{x})$ is near-standard if ERNA proves

$$
(\forall \vec{x})\left(\forall \omega^{\prime}, \omega^{\prime \prime}\right)\left(\tau\left(\omega^{\prime}, \vec{x}\right) \approx \tau\left(\omega^{\prime \prime}, \vec{x}\right)\right) .
$$

An atomic inequality $\tau(\omega, \vec{x}) \leq \sigma(\omega, \vec{x})$ is called near-standard if both members are. Since $x=y$ is equivalent to $x \leq y \wedge x \geq y$, and $\mathcal{N}(x)$ to $\lceil x\rceil=|x|$, any internal 
formula $\varphi(\omega, \vec{x})$ can be assumed to consist entirely of atomic inequalities; it is called near-standard if it is made up of near-standard atomic inequalities.

An example of a near-standard term is $\arctan x:=\int_{0}^{\underline{x}} \frac{1}{1+t^{2}} d_{\pi_{1}} t$, where $\pi_{1}$ is a fixed infinitely fine partition (see definitions 17,18 and 28 below). Defining $\pi:=4 \sum_{k=0}^{\omega} \frac{(-1)^{k}}{2 k+1}$, the following formula is near-standard:

$$
x>0 \rightarrow \arctan x \leq \frac{\pi}{2} .
$$

Another example is $\sqrt{x}$ which satisfies $(\sqrt{x})^{2} \approx x$ for $x \in[0,1]$. This 'square root function' is defined as $\sqrt{x}:=(\mu n \leq \omega)\left[\left(\frac{n}{\omega}\right)^{2} \geq x\right] / \omega$ (see [11, Example 5.2.4] and [12, Theorem 58]). The following formula is near-standard:

$$
0<x<y^{2}<1 \rightarrow \sqrt{x}<y .
$$

In stronger theories of Nonstandard Analysis, near-standard terms such as arctan $x$ and $\sqrt{x}$, defined above, would be converted to standard terms by the 'standard part map' st $(x)$ which satisfies $\operatorname{st}(x+\varepsilon)=x$, for $\varepsilon \approx 0$ and standard $x$. However, ERNA does not have such a map and hence functions of basic analysis like $\arctan x$ and $\sqrt{x}$, defined above, are not allowed in $\Pi_{1}$-TRANS. Moreover, full transfer for near-standard formulas is actually impossible: formulas (2) and (3) are true for all rational $x, y$, but there are hyperrational counterexamples. Nonetheless, the following formulas hold for all $x, y$

$$
x>0 \rightarrow \arctan x \lesssim \frac{\pi}{2} \text { and } 0<x<y^{2}<1 \rightarrow \sqrt{x} \lesssim y .
$$

Replacing ' $\leq$ ' with ' $\lesssim$ ' for near-standard formulas is the key idea behind generalising the scope of transfer, as is clear from definition 6 and (5) below. However, we need a few definitions, first 'positive' and 'negative' occurrence of subformulas (see [5, p. 15]).

Intuitively speaking, an occurrence of a subformula $B$ in $A$ is positive (negative) if, after resolving the implications outside $B$ and pushing all negations inward, but not inside $B$, there is no (one) negation in front of $B$. Thus, in

$$
(\neg(B \rightarrow C) \wedge(D \rightarrow B)) \rightarrow \neg D,
$$

all occurrences of $B$ are negative, $C$ has one positive occurrence and $D$ occurs both positively and negatively. The formal definition is as follows.

4. Definition. Given a formula $A$, an occurrence of a subformula $B$, and an occurrence of a logical connective $\alpha$ in $A$, we say that $B$ is negatively bound by $\alpha$ if either $\alpha$ is a negation $\neg$ and $B$ is in its scope, or $\alpha$ is an implication $\rightarrow$ and $B$ is a subformula of the antecedent. The subformula $B$ is said to occur negatively (positively) in $A$ if $B$ is negatively bound by an odd (even) number of connectives of $A$.

5. Notation. We write $a \ll b$ for $a \leq b \wedge a \not b$ and $a \lesssim b$ for $a \leq b \vee a \approx b$.

6. Definition. Given a near-standard formula $\varphi(\vec{x})$, let $\bar{\varphi}(\vec{x})$ be the formula obtained by replacing every positive (negative) occurrence of a near-standard inequality $\leq$ with $\lesssim(\ll)$.

Now consider the following transfer principles. The first one is ERNA's transfer principle for universal formulas (see [12, Axiom schema 43]).

7. Principle $\left(\Pi_{1}\right.$-TRANS). Let $\varphi(x)$ be standard and quantifier-free. Then

$$
\left(\forall^{s t} x\right) \varphi(x) \rightarrow(\forall x) \varphi(x) .
$$

8. Principle $\left(\bar{\Pi}_{1}-\mathrm{TRANS}\right)$. Let $\varphi(x)$ be near-standard and quantifier-free. Then

$$
\left(\forall^{s t} x\right) \varphi(x) \rightarrow(\forall x) \bar{\varphi}(x) \text {. }
$$


The second principle is also called 'bar transfer'. When formulated in contrapositive form, bar transfer is called ' $\bar{\Sigma}_{1}$-TRANS'. Although bar transfer has a much wider scope than $\Pi_{1}$-transfer, the two are in fact equivalent.

9. Theorem. In ERNA, the schemas $\Pi_{1}$-TRANS and $\bar{\Pi}_{1}$-TRANS are equivalent.

Proof. For a standard formula, there holds $\bar{\varphi} \equiv \varphi$ and hence the schema $\bar{\Pi}_{1}$-TRANS clearly implies $\Pi_{1}$-TRANS. Now assume $\Pi_{1}$-TRANS, let $\varphi$ be as in $\bar{\Pi}_{1}$-TRANS and let $\tau_{1}$ and $\tau_{2}$ be near-standard terms. We first prove the atomic case, i.e. where $\varphi(n)$ is $\tau_{1}(n, \omega) \leq \tau_{2}(n, \omega)$. So, assume that $\varphi(n)$ holds for all $n \in \mathbb{N}$, and consider the sentence

$$
(\forall n)\left(\forall \omega^{\prime}, \omega^{\prime \prime}\right)\left(\tau_{i}\left(n, \omega^{\prime}\right) \approx \tau_{i}\left(n, \omega^{\prime \prime}\right)\right)
$$

for $i=1,2$. This sentence expresses that $\tau_{1}$ and $\tau_{2}$ are near-standard. Also, it implies

and also

$$
\left(\forall^{s t} k\right)(\forall n)\left(\forall \omega^{\prime}, \omega^{\prime \prime}\right)\left(\left|\tau_{i}\left(n, \omega^{\prime}\right)-\tau_{i}\left(n, \omega^{\prime \prime}\right)\right|<1 / k\right)
$$

$$
\left(\forall^{s t} k\right)\left(\forall \omega^{\prime}, \omega^{\prime \prime}\right)\left(\forall n \leq \omega_{1}\right)\left(\left|\tau_{i}\left(n, \omega^{\prime}\right)-\tau_{i}\left(n, \omega^{\prime \prime}\right)\right|<1 / k\right),
$$

where $\omega_{1}$ is a fixed infinite hypernatural number. By underflow ([12, Theorem 54]),

$$
\left(\forall^{s t} k\right)\left(\exists^{s t} M\right)\left(\forall m, m^{\prime} \geq M\right)\left(\forall n \leq \omega_{1}\right)\left(\left|\tau_{i}(n, m)-\tau_{i}\left(n, m^{\prime}\right)\right|<1 / k\right)
$$

and $\Pi_{1}$-transfer implies

$$
\left(\forall^{s t} k\right)\left(\exists^{s t} M\right)\left(\forall m, m^{\prime} \geq M\right)(\forall n)\left(\left|\tau_{i}(n, m)-\tau_{i}\left(n, m^{\prime}\right)\right|<1 / k\right) .
$$

Now suppose there exists a number $n_{0}$ satisfying $\tau_{1}\left(n_{0}, \omega\right) \gg \tau_{2}\left(n_{0}, \omega\right)$ and assume $k_{0} \in \mathbb{N}$ is such that $\tau_{1}\left(n_{0}, \omega\right)-\tau_{2}\left(n_{0}, \omega\right)>1 / k_{0}$. Then apply $(9)$ for $k=4 k_{0}$ and obtain, for $i=1,2$, a number $M_{i} \in \mathbb{N}$ such that

$$
(\forall n)\left(\forall m, m^{\prime} \geq M_{i}\right)\left(\left|\tau_{i}(n, m)-\tau_{i}\left(n, m^{\prime}\right)\right|<1 / 4 k_{0}\right) .
$$

In particular, this implies

$$
\left|\tau_{i}\left(n_{0}, M_{i}\right)-\tau_{i}\left(n_{0}, \omega\right)\right|<1 / 4 k_{0},
$$

for $i=1,2$. This formula, together with $\tau_{1}\left(n_{0}, \omega\right)-\tau_{2}\left(n_{0}, \omega\right)>1 / k_{0}$, implies

$$
\tau_{1}\left(n_{0}, M_{1}\right)-\tau_{2}\left(n_{0}, M_{2}\right)>1 / 2 k_{0},
$$

yielding

By $\Sigma_{1}$-transfer, we obtain

$$
(\exists n)\left(\tau_{1}\left(n, M_{1}\right)-\tau_{2}\left(n, M_{2}\right)>1 / 2 k_{0}\right) .
$$

$$
\left(\exists^{s t} n\right)\left(\tau_{1}\left(n, M_{1}\right)-\tau_{2}\left(n, M_{2}\right)>1 / 2 k_{0}\right) .
$$

By 10 , this implies

$$
\left(\exists^{s t} n\right)\left(\tau_{1}(n, \omega)-\tau_{2}(n, \omega)>0\right),
$$

which contradicts our assumption $\left(\forall^{s t} n\right)\left(\tau_{1}(n, \omega) \leq \tau_{2}(n, \omega)\right)$.

For the general case, we use induction on the number of near-standard atomic formulas. We may assume that in $\bar{\varphi}$ each instance of $A \rightarrow B$ is replaced by $\neg A \vee B$ and that all negations have been pushed in front of the atomic formulas by using De Morgan's laws from left to right. By definition, each instance of $a \ll b$ in $\bar{\varphi}$ occurs negatively and hence each instance of $a \ll b$ now occurs as $\neg(a \ll b)$. Thus, it can be replaced by $a \gtrsim b$ and hence we may assume $\bar{\varphi}$ to be free of ' $\ll$ '.

In case only one near-standard atomic formula occurs in $\varphi(n)$, the latter has the form either $\tau_{1}(n, \omega) \leq \tau_{2}(n, \omega) \wedge \psi(n)$ or $\tau_{1}(n, \omega) \leq \tau_{2}(n, \omega) \vee \psi(n)$, with $\psi \in L^{s t}$ quantifier-free. In the first case, consider $\left(\forall^{s t}\right) \varphi(n)$ and push the universal quantifier through the conjunction. Now apply regular $\Pi_{1}$-transfer to the second part of the conjunction and apply the atomic case treated above to the first part. Hence there follows $(\forall n) \bar{\varphi}(n)$. For the second case, assume $\left(\forall^{s t} n\right) \varphi(n)$ and suppose there is a 
number $n_{0}$ such that $\neg \bar{\varphi}\left(n_{0}\right)$, i.e. $\tau_{1}\left(n_{0}, \omega\right) \gg \tau_{2}\left(n_{0}, \omega\right) \wedge \neg \psi\left(n_{0}\right)$. In the exactly the same way as in the atomic case above, we obtain (10) and (11). As there also holds $\neg \psi\left(n_{0}\right)$, 111 implies

$$
(\exists n)\left[\tau_{1}\left(n, M_{1}\right)-\tau_{2}\left(n, M_{2}\right)>1 / 2 k_{0} \wedge \neg \psi(n)\right] .
$$

The previous formula is standard and hence, by $\Sigma_{1}$-transfer, there follows

$$
\left(\exists^{s t} n\right)\left[\tau_{1}\left(n, M_{1}\right)-\tau_{2}\left(n, M_{2}\right)>1 / 2 k_{0} \wedge \neg \psi(n)\right] .
$$

By 10 , there follows

$$
\left(\exists^{s t} n\right)\left[\tau_{1}(n, \omega)-\tau_{2}(n, \omega)>0 \wedge \neg \psi(n)\right] .
$$

This contradicts our assumption that $\varphi(n)$ holds for all $n \in \mathbb{N}$ and this case is done.

Now assume we have established the case for $m \geq 1$ occurrences of near-standard atomic formulas. We now prove bar transfer for formulas $\varphi(n)$ with $m+1$ occurrences of near-standard atomic formulas. Again, the formula $\varphi(n)$ has the form $\tau_{1}(n, \omega) \leq \tau_{2}(n, \omega) \wedge \psi(n)$ or $\tau_{1}(n, \omega) \leq \tau_{2}(n, \omega) \vee \psi(n)$, where $\psi$ has only $m$ occurrences of near-standard atomic formulas. The first case is treated in the same way as in the case for $m=1$, with the exception that the induction hypothesis is invoked to apply bar transfer to $\left(\forall^{s t} n\right) \psi(n)$. For the second case, assume $\left(\forall^{s t} n\right) \varphi(n)$ and suppose there is a number $n_{0}$ such that $\neg \bar{\varphi}\left(n_{0}\right)$, i.e. $\tau_{1}\left(n_{0}, \omega\right) \gg \tau_{2}\left(n_{0}, \omega\right) \wedge \neg \bar{\psi}\left(n_{0}\right)$. In the same way as before, we obtain (10) and (11). As there also holds $\neg \bar{\psi}\left(n_{0}\right)$, (11) implies

$$
(\exists n)\left[\tau_{1}\left(n, M_{1}\right)-\tau_{2}\left(n, M_{2}\right)>1 / 2 k_{0} \wedge \neg \bar{\psi}(n)\right] .
$$

The previous formula only involves $m$ occurrences of atomic near-standard formulas and hence the induction hypothesis applies to it. By $\bar{\Sigma}_{1}$-transfer, there follows

$$
\left(\exists^{s t} n\right)\left[\tau_{1}\left(n, M_{1}\right)-\tau_{2}\left(n, M_{2}\right)>1 / 2 k_{0} \wedge \neg \psi(n)\right] .
$$

By 100 , there follows

$$
\left(\exists^{s t} n\right)\left[\tau_{1}(n, \omega)-\tau_{2}(n, \omega)>0 \wedge \neg \psi(n)\right] .
$$

This contradicts our assumption that $\varphi(n)$ holds for all $n \in \mathbb{N}$ and we are done.

Note that it is clear from the proof of the atomic case why we cannot weaken the predicate ' $\ll$ ' or strengthen the predicate 'ミ) in bar transfer. Also, without theorem 9 all items listed in theorem 2 would be limited to standard functions. This would exclude most functions from basic analysis, like ERNA's cosine and exponential.

\section{Mathematics in ERNA}

3.1. Mathematics without Transfer. In this section, we obtain some well-known mathematical results in the theory ERNA. The 'running theme' is that ERNA can prove many theorems of ordinary mathematics, as long as we allow an infinitesimal error. This theme is best expressed in theorems 12, 16, 29, and 31. Note that every theorem in this section is proved in ERNA.

Hereafter, we assume that $a$ and $b$ are finite numbers such that $a \not \approx b$. A function $f(x)$ is an internal term, not involving min and everywhere defined. Also, we recall ERNA's weight function $\|x\|$ which is defined as $\| \pm n / m\|=\max \{n, m\}$, for hypernatural $n, m$ with $m \neq 0$ (see [12, Theorem 23]). The following theorem is used throughout this paper.

10. Theorem. In ERNA, there are hyperrationals of arbitrarily large weight between any two numbers. 
Proof. If $a$ is a hyperrational, $\|-a\|=\|a\|$ and $\|1 / a\|=\|a\|$ if $a \neq 0$. Hence we can restrict ourselves to given hyperrationals $1 \leq a<b$. Write $a=\frac{a_{1}}{a_{2}}$ with $a_{1}$ and $a_{2}$ relatively prime hypernaturals. From $a \geq 1$ we deduce that $\|a\|=\max \left(a_{1}, a_{2}\right)=a_{2}$. Choose a hypernatural $n$ so large that $a<a+\frac{1}{n}<b$ and $n>a_{2}$. As Euclid's proof of the infinitude of the prime numbers can easily be formalised in ERNA, we may assume that $n$ is prime. This implies that $a_{2} n$ and $a_{1} n+a_{2}$ are relatively prime. Indeed, $n$ is not a common divisor, as it would divide $a_{2}<n$. Therefore, a common divisor $d>1$ would divide $a_{2}$, hence also $\left(a_{1} n+a_{2}\right)-a_{2}$ and finally $a_{1}$. Therefore

$$
\left\|a+\frac{1}{n}\right\|=\left\|\frac{a_{1} n+a_{2}}{a_{2} n}\right\|=\max \left(a_{1} n+a_{2}, a_{2} n\right)=a_{1} n+a_{2}=\|a\| n+a_{2},
$$

growing arbitrarily large with $n$

3.1.1. Continuity. First, we introduce the notion of (nonstandard) continuity in ERNA and prove some fundamental results.

11. Definition. A function $f(x)$ is 'continuous over $[a, b]$ ' if

$$
(\forall x, y \in[a, b])(x \approx y \rightarrow f(x) \approx f(y)) .
$$

The attentive reader has noted that we work with the nonstandard version of uniform continuity. There are two reasons for this. First of all, if we limit the variable $x$ in 14 to $\mathbb{Q}$, the function $\frac{1}{x^{2}-2}$ satisfies the resulting formula, although this function is infinite in the interval $[-2,2]$. Similarly, the function $g(x)$, defined as 1 if $x^{2}<2$ and 0 if $x^{2} \geq 2$, satisfies (14) with $x$ limited to $\mathbb{Q}$, but this function has a jump in its graph. The same holds for the pointwise $\varepsilon-\delta$ continuity and thus both are not suitable for our purposes. Second, in light of theorem 2 , the $\varepsilon-\delta$ definition of uniform continuity is closely related to $\Pi_{1}$-transfer. In the absence of the latter principle, we are left with 114 .

12. Theorem (Weierstrass extremum theorem). If $f$ is continuous over $[a, b]$, there is a number $c \in[a, b]$ such that for all $x \in[a, b]$, we have $f(x) \lesssim f(c)$.

Proof. Let $a, b, f$ be as stated. The points $x_{n}=a+\frac{n(b-a)}{\omega}$, for hypernatural $1 \leq n \leq \omega-1$, partition the interval $[a, b]$ in infinitesimal subintervals $\left[x_{n}, x_{n+1}\right]$. Every $x \in[a, b]$ is in one of these intervals, hence infinitely close to both of its end points. As $f$ is continuous over $[a, b]$, we have $f(x) \approx f\left(x_{n}\right)$ for $x \in\left[x_{n}, x_{n+1}\right]$. ERNA has an explicit maximum operator, see [12, Section 5.1] and we define

$$
M:=\max _{0 \leq n \leq \omega-1} f\left(x_{n}\right) .
$$

Hence $f(x) \lesssim M$ for all $x \in[a, b]$.

13. Corollary. If $f$ is continuous on $[a, b]$ and finite in at least one point, then it is finitely bounded on $[a, b]$.

Proof. Let $a, b, f$ be as stated. Denote by $\varphi(n)$ the formula

$$
(\forall x, y \in[a, b])(|x-y| \leq 1 / n \wedge\|x, y\| \leq \omega \rightarrow|f(x)-f(y)|<1) .
$$

As $f$ is continuous, this formula holds for all infinite $n$. By [12, Corollary 53], (16) may be treated as quantifier-free. Underflow yields that it holds from some finite $n_{0}$ on. Assume $f\left(x_{0}\right)$ is finite in $x_{0} \in[a, b]$. Partitioning $[a, b]$ with points $1 / \omega$ apart shows that we may assume $\left\|x_{0}\right\| \leq \omega$. From $\varphi(n)$ for $n=n_{0}$, it easily follows that $f(c)$ given by the theorem deviates at most $n_{0}\lceil b-a\rceil$ from $f\left(x_{0}\right)$.

14. Corollary. If $f$ is near-standard and cont. on $[a, b]$, it is finitely bounded there.

Proof. From (1), we can derive (8) for $f(x, \omega)$ instead of $\tau_{i}(n, \omega)$. Thus $f$ is finitely close to a standard term in at least one point. By [12, Theorem 30], this standard term is finite and hence $f$ is finite in at least one point. 
15. Theorem (Intermediate value theorem). If $f$ is continuous on $[a, b]$, and $f(a) \leq$ $y_{0} \leq f(b)$, then there is an $x_{0} \in[a, b]$ such that $f\left(x_{0}\right) \approx y_{0}$.

Proof. Let $a, b, y_{0}$ and $f$ be stated. The points $x_{n}=a+\frac{n(b-a)}{\omega}$, for hypernatural $1 \leq n \leq \omega-1$, partition the interval $[a, b]$ in infinitesimal subintervals $\left[x_{n}, x_{n+1}\right]$. Similarly, the points $f\left(x_{n}\right)$ partition the interval $[f(a), f(b)]$ in subintervals. As $f$ is continuous, the intervals $\left[f\left(x_{n}\right), f\left(x_{n+1}\right)\right]$ are also infinitesimal. Using ERNA's explicit 'least' operator (see [12, Section 5.1]), we find an $N \leq \omega$ such that $\mid y_{0}-$ $f\left(x_{N}\right) \mid$ is minimal. Hence we have $y_{0} \in\left[f\left(x_{N}\right), f\left(x_{N+1}\right)\right]$ or $y_{0} \in\left[f\left(x_{N-1}\right), f\left(x_{N}\right)\right]$. In either case, $x_{0}=x_{N}$ satisfies the requirements.

Note that if there are rational $x_{1}$ and $y_{1}$ such that $x_{0} \approx x_{1}$ and $y_{0} \approx y_{1}$, then $y_{0} \approx f\left(x_{0}\right)$ implies $y_{1}=f\left(x_{1}\right)$, if $f$ is continuous. Most numbers in ERNA, however, do not have a standard number infinitely close.

The previous theorem includes the one-dimensional Brouwer fixed point theorem.

16. Corollary (Brouwer fixed point theorem). If $f:[0,1] \rightarrow[0,1]$ is continuous, then there is an $x_{0} \in[0,1]$ such that $f\left(x_{0}\right) \approx x_{0}$.

Proof. Let $f$ be as stated. If $f(0) \approx 0$ or $f(1) \approx 1$, we are done. Otherwise, $f(1)-1 \ll 0$ and $f(0)-0 \gg 0$. Applying the theorem to the function $f(x)-x$, we find $x_{0}$ such that $f\left(x_{0}\right)-x_{0} \approx 0$.

Note that the theorem and the corollary also follow if $f$ only satisfies (14) for $x$ and $y$ of weight at most some infinite $\omega_{1}$.

3.1.2. Riemann integration. The next step in the construction of elementary calculus is the Riemann integral. In Darboux's approach, a function is integrable if the infimum of the upper sums equals the supremum of the lower sums. Although several supremum principles are provable in ERNA and its extensions (see [12]), they are not very suited for a Darboux-like integral, because the supremum of nonstandard objects like lower sums does not have sufficiently strong properties. Therefore, we adopt Riemann's original approach, defining the integral as the limit of Riemann sums over ever finer partitions.

17. Definition. In ERNA, a partition $\pi$ of $[a, b]$ is a vector $\left(x_{1}, \ldots, x_{n+1}, t_{1}, \ldots t_{n}\right)$ such that $x_{i} \leq t_{i} \leq x_{i+1}$ for all $1 \leq i \leq n$ and $a=x_{1}$ and $b=x_{n+1}$. The number $\delta_{\pi}=\max _{1 \leq i \leq n}\left(x_{i+1}-x_{i}\right)$ is called the 'mesh' of the partition $\pi$. We call a partition 'infinitely fine' if its mesh is infinitesimal.

Riemann integration implies quantifying over all partitions of an interval, which, as such, is not a first order-operation. However, encoding hyperfinite sets to hypernatural numbers, we are left with quantifying over all hypernaturals. The pairing function defined in [12, Section 5.2] is not suited for that purpose, because its iterations grow too fast for ERNA. Instead, we will use the pairing function

$$
\pi^{(2)}(x, y)=\frac{1}{2}(x+y)(x+y+1)+y \text {. }
$$

All its iterations

$$
\pi^{(n)}\left(x_{1}, \ldots x_{n}\right):=\pi\left(\pi^{(n-1)}\left(x_{1}, \ldots, x_{n-1}\right), x_{n}\right) .
$$

are available in ERNA, as one readily verifies by induction that

$$
\left\|\pi^{(n)}\left(x_{1}, \ldots, x_{n}\right)\right\| \leq 2^{2^{n+1}}\left\|x_{1}, \ldots, x_{n}\right\|^{2^{n}},
$$

for all $x_{i}$ and hypernatural $n>2$. Similarly, the decoding function $\left(\pi^{(n)}\right)^{-1}$, which yields the vector $\left(x_{1}, \ldots, x_{n}\right)$ when applied to $\pi^{(n)}\left(x_{1}, \ldots, x_{n}\right)$, can be defined in ERNA. Thus, ERNA allows quantification over all partitions of an interval. 
18. Definition (Riemann Integration). Let $f$ be a function defined on $[a, b]$.

(1) The Riemann sum corresponding to a partition $\left(x_{1}, \ldots, x_{n+1}, t_{1}, \ldots, t_{n}\right)$ is defined as $\sum_{i=1}^{n} f\left(t_{i}\right)\left(x_{i+1}-x_{i}\right)$.

(2) The function $f$ is called 'Riemann integrable on $[a, b]$ ' if Riemann sums of infinitely fine partitions are finite and infinitely close to each other. If so, the Riemann sum corresponding to the infinitely fine partition $\pi$ of $[a, b]$ is denoted by $\int_{a}^{b} f(x) d_{\pi} x$.

19. Theorem. A function, continuous and finite over $[a, b]$, is Riemann integrable over that interval.

Proof. Let $f$ be as stated and consider two infinitely fine partitions $\pi_{1}$ and $\pi_{2}$ of $[a, b]$. Let $\sum_{i=1}^{\omega_{1}} f\left(t_{i}\right)\left(x_{i+1}-x_{i}\right)$ and $\sum_{i=1}^{\omega_{2}} f\left(s_{i}\right)\left(y_{i+1}-y_{i}\right)$ be the respective Riemann sums. Using ERNA's definition by cases (see [12, Corollary 51]), we modify $\pi_{1}$ in the following way: if $\left[x_{i}, x_{i+1}\right]$ contains some $y_{j}$, break it into subintervals $\left[x_{i}, y_{j}\right]$ and $\left[y_{j}, x_{i+1}\right]$ and rename these subintervals to $\left[z_{i}, z_{i+1}\right]$ and $\left[z_{i+1}, z_{i+2}\right]$. Thus, the entry $f\left(t_{i}\right)\left(x_{i+1}-x_{i}\right)$ of the Riemann sum of $\pi_{1}$ is replaced by $f\left(t_{i+1}^{\prime}\right)\left(z_{i+2}-\right.$ $\left.z_{i+1}\right)+f\left(t_{i}^{\prime}\right)\left(z_{i+1}-z_{i}\right)$ with $t_{i+1}^{\prime}:=t_{i}$ and $t_{i}^{\prime}:=t_{i}$. Proceeding in the same way for $\pi_{2}$, we convert the original Riemann sums into $\sum_{i=1}^{\omega_{3}} f\left(t_{i}^{\prime}\right)\left(z_{i+1}-z_{i}\right)$ and $\sum_{i=1}^{\omega_{3}} f\left(s_{i}^{\prime}\right)\left(z_{i+1}-z_{i}\right)$, which share the upper bound $\omega_{3}$ and the partition points. As all indices $i$ and $j$ are bounded by $\omega_{1}+\omega_{2}+2$, this procedure is compatible with ERNA's definition by cases. Also, by construction, $t_{i}^{\prime} \approx s_{i}^{\prime}$. Hence we have

$$
\begin{aligned}
\sum_{i=1}^{\omega_{1}} f\left(t_{i}\right)\left(x_{i+1}-x_{i}\right) & -\sum_{i=1}^{\omega_{2}} f\left(s_{i}\right)\left(y_{i+1}-y_{i}\right) \\
& =\sum_{i=1}^{\omega_{3}} f\left(t_{i}^{\prime}\right)\left(z_{i+1}-z_{i}\right)-\sum_{i=1}^{\omega_{3}} f\left(s_{i}^{\prime}\right)\left(z_{i+1}-z_{i}\right) \\
& =\sum_{i=1}^{\omega_{3}}\left(f\left(t_{i}^{\prime}\right)-f\left(s_{i}^{\prime}\right)\right)\left(z_{i+1}-z_{i}\right) .
\end{aligned}
$$

Let $\bar{\varepsilon}$ be the maximum of the $\left|f\left(t_{i-1}^{\prime}\right)-f\left(s_{i-1}^{\prime}\right)\right|$ for $2 \leq i \leq \omega_{3}$, as provided by ERNA's explicit max operator. Because $f$ is continuous over $[a, b]$, we have $\bar{\varepsilon} \approx 0$ and so the absolute value of $(17)$ is at most $\sum_{n=1}^{\omega_{3}} \bar{\varepsilon}\left(z_{i}-z_{i-1}\right)=\bar{\varepsilon}(b-a) \approx 0$. This implies that the Riemann sums considered are infinitely close to each other. By theorem 12 , the function $f$ is bounded on $[a, b]$ and hence every Riemann sum is in absolute value at most the finite number $(M+1)(b-a)$, with $M$ as in $(15)$.

3.1.3. Differentiation. Another key element of analysis is the derivative, defined in this paragraph. For brevity, we write ' $\Delta_{h} f(x)$ ' for $\frac{f(x+h)-f(x)}{h}$.

20. Definition. [Differentiability] A function $f$ is 'differentiable over $(a, b)$ ' if $\Delta_{\varepsilon} f(x) \approx$ $\Delta_{\varepsilon^{\prime}} f(x)$ is finite for all nonzero $\varepsilon, \varepsilon^{\prime} \approx 0$ and all $a \ll x \ll b$.

If $f$ is differentiable over $(a, b)$ and $0 \neq \varepsilon \approx 0$, then $\Delta_{\varepsilon} f\left(x_{0}\right)$ is called the ' $\varepsilon$ derivative of $f$ at $x_{0}$ ' and denoted by $f_{\varepsilon}^{\prime}\left(x_{0}\right)$. Any $f_{\varepsilon}^{\prime}\left(x_{0}\right)$ with nonzero $\varepsilon \approx 0$ is a representative of 'the' derivative $f^{\prime}\left(x_{0}\right)$, which is only defined up to infinitesimals. Thus, any statement about $f^{\prime}\left(x_{0}\right)$ should be interpreted as a statement about $\Delta_{\varepsilon} f\left(x_{0}\right)$, quantified over all nonzero $\varepsilon \approx 0$.

A weaker notion than differentiability is provided by

21. Definition. [S-differentiability] A function $f$ is called 'S-differentiable over $(a, b)^{\prime}$ if $\Delta_{\varepsilon} f(x) \approx \Delta_{\varepsilon^{\prime}} f(x)$ is finite for all large enough $\varepsilon, \varepsilon^{\prime} \approx 0$ and all $a \ll x \ll b$. 
The informal expression 'for all large enough infinitesimals' in the previous definition is short for the $\Sigma_{2}$-statement

$$
\begin{aligned}
\left(\exists \varepsilon_{0} \approx 0\right) & \left(\forall \varepsilon, \varepsilon^{\prime} \approx 0\right)(\forall x) \\
& {\left[a \ll x \ll b \wedge\left|\varepsilon_{0}\right|<|\varepsilon|,\left|\varepsilon^{\prime}\right| \rightarrow \Delta_{\varepsilon} f\left(x_{0}\right) \approx \Delta_{\varepsilon^{\prime}} f\left(x_{0}\right)\right] . }
\end{aligned}
$$

The derivative is defined in the same way as for definition 20. A crucial point is that $\varepsilon_{0}$ does not depend on the choice of $x$. Indeed, otherwise $\varepsilon_{0}$ would be a function of $x$, i.e. in $(18)$ the quantifier ' $(\forall x)$ ' would be at the front. However, in ERNA, it is not possible to compute the function $\varepsilon_{0}(x)$ from the latter formula, as it involves ' $\approx$ '. In this case, the derivative would not be defined properly as it cannot be computed in a straightforward way.

Furthermore, 'S-differentiable' is short for 'standardly differentiable', and it does imply the classical $\varepsilon-\delta$-definition of uniform differentiability, as we show in the next theorem. Thus, as in the case of continuity, the uniform version of differentiability is more natural than the pointwise one. Such phenomenon also occurs in the setting of Constructive Mathematics. The latter is briefly considered in section 4. A more utilitarian argument in favour of S-differentiability is that it arises naturally in the proof of ERNA's version of the first fundamental theorem of calculus and Peano's existence theorem.

22. Theorem. If $f$ is $S$-differentiable over $(a, b)$, we have that

$$
\begin{aligned}
\left(\forall^{s t} k\right)\left(\exists^{s t} N\right) & \left(\forall^{s t} h, h^{\prime}\right)\left(\forall^{s t} x \in(a, b)\right) \\
& {\left[0<|h|,\left|h^{\prime}\right|<1 / N \rightarrow\left|\Delta_{h} f(x)-\Delta_{h^{\prime}} f(x)\right|<1 / k\right] . }
\end{aligned}
$$

Proof. Choose $\varepsilon_{0}$ as in (18) and fix an infinite hypernatural $\omega_{0}$. We see that

$$
\left(\forall h, h^{\prime}\right)(\forall x \in(a, b))\left(\left|\varepsilon_{0}\right|<|h|,\left|h^{\prime}\right| \leq 1 / \omega \wedge\left\|h, h^{\prime}, x\right\| \leq \omega_{0} \rightarrow \Delta_{h} f(x) \approx \Delta_{h^{\prime}} f(x)\right)
$$

holds for all infinite hypernatural $\omega$. Fixing $k \in \mathbb{N}$, we have in particular

$\left(\forall h, h^{\prime}\right)(\forall x \in(a, b))\left(\left|\varepsilon_{0}\right|<|h|,\left|h^{\prime}\right| \leq 1 / \omega \wedge\left\|h, h^{\prime}, x\right\| \leq \omega_{0} \rightarrow\left|\Delta_{h} f(x)-\Delta_{h^{\prime}} f(x)\right|<\frac{1}{k}\right)$,

for all infinite hypernatural $\omega$. By [12, Corollary 53], this formula is equivalent to a quantifier-free one and underflow yields

$\left(\forall h, h^{\prime}\right)(\forall x \in(a, b))\left(\left|\varepsilon_{0}\right|<|h|,\left|h^{\prime}\right| \leq 1 / N \wedge\left\|h, h^{\prime}, x\right\| \leq \omega_{0} \rightarrow\left|\Delta_{h} f(x)-\Delta_{h^{\prime}} f(x)\right|<\frac{1}{k}\right)$, for all $N \geq N(k) \in \mathbb{N}$, implying $(19)$.

Since $S$-differentiability is stronger than 'normal' differentiability, the derivative will have stronger properties, as witnessed by the following theorem. A function is said to be 'continuous over $(a, b)$ ' if it satisfies 14 for all $a \ll x, y \ll b$.

23. Theorem. If $f$ is differentiable over $(a, b)$, then $f^{\prime}(x)$ is cont. over $(a, b)$.

Proof. Choose two points $x \approx y$ such that $a \ll x<y \ll b$. If $y-x=\varepsilon$, then

$$
\Delta_{\varepsilon} f(x)=\frac{f(x+\varepsilon)-f(x)}{\varepsilon}=\frac{f(y)-f(y-\varepsilon)}{\varepsilon}=\frac{f(y-\varepsilon)-f(y)}{-\varepsilon}=\Delta_{-\varepsilon} f(y) \approx \Delta_{\varepsilon} f(y),
$$

and thus $f_{\varepsilon^{\prime}}^{\prime}(x) \approx f_{\varepsilon}^{\prime}(x) \approx f_{\varepsilon}^{\prime}(y) \approx f_{\varepsilon^{\prime}}^{\prime}(y)$, for all nonzero $\varepsilon^{\prime} \approx 0$.

The theorem generalizes to S-differentiable functions, but not in an elegant way.

24. Corollary. If $f$ is $S$-differentiable over $(a, b)$, then $f_{\varepsilon}^{\prime}(x)$ is continuous over $(a, b)$, for $\varepsilon \approx 0$ large enough.

Proof. Let $\varepsilon_{0}>0$ be as in $[18$. Choose points $x \approx y$ such that $a \ll x<y \ll b$. First, suppose $y-x=\varepsilon \geq \varepsilon_{0}$. The same proof as in the theorem yields this case. Now suppose $y-x=\varepsilon<\varepsilon_{0}$ and define $z=y+2 \varepsilon_{0}$. Then $z-x=\varepsilon^{\prime} \geq \varepsilon_{0}$ and $z-y=\varepsilon^{\prime \prime} \geq \varepsilon_{0}$ and by the previous case, we have $f_{\varepsilon^{\prime}}^{\prime}(x) \approx f_{\varepsilon^{\prime}}^{\prime}(z)$ and 
$f_{\varepsilon^{\prime \prime}}^{\prime}(z) \approx f_{\varepsilon^{\prime \prime}}^{\prime}(y)$. By the definition of S-differentiability, we have $f_{\varepsilon^{\prime}}^{\prime}(z) \approx f_{\varepsilon^{\prime \prime}}^{\prime}(z)$, and thus $f_{\varepsilon^{\prime \prime \prime}}^{\prime \prime}(x) \approx f_{\varepsilon^{\prime}}^{\prime}(x) \approx f_{\varepsilon^{\prime \prime}}^{\prime}(y) \approx f_{\varepsilon^{\prime \prime \prime}}^{\prime}(y)$, for all $\varepsilon^{\prime \prime \prime} \geq \varepsilon_{0}$.

Since the derivative is only defined up to infinitesimals in ERNA, the statement $f^{\prime}(x)>0$ is not very strong, as $f^{\prime}(x) \approx 0$ may hold at the same time. Similarly, $f(x)<f(y)$ is consistent with $f(x) \approx f(y)$ and we need stronger forms of inequality to express meaningful properties of functions and their derivatives.

25. Definition. A function $f$ is strictly increasing over an interval $[a, b]$, if for all $x, y \in[a, b]$ we have $x \ll y \rightarrow f(x) \ll f(y)$.

26. Theorem. If $f$ is differentiable over $(a, b)$, there is an $N \in \mathbb{N}$ such that

(1) if $f^{\prime}\left(x_{0}\right) \gg 0$, then $f$ is strictly increasing in $\left[x_{0}-\frac{1}{N}, x_{0}+\frac{1}{N}\right]$,

(2) if $f^{\prime}\left(x_{0}\right) \ll 0$, then $f$ is strictly decreasing in $\left[x_{0}-\frac{1}{N}, x_{0}+\frac{1}{N}\right]$.

Proof. For the first item, $f^{\prime}(x) \gg 0$ implies $f(y)>f(z)$ for all $y, z$ satisfying $y, z \approx x_{0}$ and $y>z$. Fix an infinite number $\omega_{1}$ and let $M \gg 0$ be $f^{\prime}\left(x_{0}\right) / 2$. By the previous observation, the following sentence is true for all infinite hypernaturals $\omega$

$(\forall y, z)\left[\|y, z\| \leq \omega_{1} \wedge y>z \wedge\left|x_{0}-z\right|<\frac{1}{\omega} \wedge\left|x_{0}-y\right|<\frac{1}{\omega} \rightarrow f(y)>f(z)+M(y-z)\right]$.

Applying overflow yields the first item, as $f$ is continuous over $(a, b)$. Likewise for the second item.

A function is said to be 'continuous in $a$ ' if 114 holds for $x=a$.

27. Theorem (Mean value theorem). If $f$ is differentiable over $(a, b)$ and continuous in $a$ and $b$, then there is an $x_{0} \in[a, b]$ such that $f^{\prime}\left(x_{0}\right) \approx \frac{f(b)-f(a)}{b-a}$.

Proof. Let $f$ be as in the theorem. First, we prove the case where $f(a) \approx f(b)$. By theorem 12, $f$ reaches its maximum (up to infinitesimals), say in $x_{0}$, and its minimum (idem), say in $x_{1}$, over $[a, b]$. If $f\left(x_{0}\right) \approx f\left(x_{1}\right) \approx f(a)$, then $f$ is constant up to infinitesimals. By theorem 26 we have $f^{\prime}(x) \approx 0$ for all $a \ll x \ll b$. If $f\left(x_{0}\right) \not \approx f(a)$, then by theorem 26 we have $f^{\prime}\left(x_{0}\right) \approx 0$. The case $f\left(x_{1}\right) \not \approx f(a)$ is treated in a similar way. The general case can be reduced to the previous case by using the function $F(x)=f(x)-\frac{f(b)-f(a)}{b-a}(x-a)$.

3.1.4. The first fundamental theorem of calculus. In this paragraph, we obtain ERNA's version of the first fundamental theorem of calculus.

28. Definition. If $\pi$ is an infinitely fine partition of $[a, b]$, we denote by $\underline{x}$ the least partition point not exceeding $x$. If $f$ is integrable over $[a, b]$, we define

$$
F_{\pi}(x):=\int_{a}^{\underline{x}} f(t) d_{\pi} t
$$

In ERNA, there is no standard-part function mapping a finite number $x$ to the unique standard number $r \approx x$. Consequently, there is no natural way to avoid that integrals are only defined up to infinitesimals. The same occurred in ERNA's predecessor $\mathrm{NQA}^{+}$proposed in [6]. There, differentiation and integration cancel each other out on the condition that the mesh $d u$ of the hyperfinite partition and the infinitesimal $y$ used in the derivative are related by $d u / y \approx 0$. This requirement is hidden under a complicated definition of the integral (see 6, Axiom 18]). Our definitions of integration and differentiation are quite natural and we still obtain an elegant version of the first Fundamental Theorem of Calculus, see corollary 30 below.

29. Theorem. Let $f$ be continuous on $[a, b]$. For every $\eta \approx 0$ and every hyperfine partition $\pi$ of $[a, b]$ with $\delta_{\pi} / \eta \approx 0$, we have $\Delta_{\eta} F_{\pi}(x) \approx f(x)$ for all $a \ll x \ll b$. 
Proof. Let $f, \pi$ and $\eta$ be as stated and fix $a \ll x_{0} \ll b$. Then we have

$$
F_{\pi}\left(x_{0}\right)=\sum_{i=1}^{\omega_{1}} f\left(t_{i}\right)\left(x_{i+1}-x_{i}\right) \text { and } F_{\pi}\left(x_{0}+\eta\right)=\sum_{n=1}^{\omega_{2}} f\left(t_{i}\right)\left(x_{i+1}-x_{i}\right),
$$

with $\omega_{2}>\omega_{1}$. Now, let $M$ be the largest and $m$ the smallest of the $f\left(t_{i}\right)$ for $\omega_{1}<i \leq \omega_{2}$. Then,

$F_{\pi}\left(x_{0}+\eta\right)-F_{\pi}\left(x_{0}\right)=\sum_{i=\omega_{1}+1}^{\omega_{2}} f\left(t_{i}\right)\left(x_{i+1}-x_{i}\right) \leq M \sum_{i=\omega_{1}+1}^{\omega_{2}}\left(x_{i+1}-x_{i}\right)=M\left(x_{\omega_{2}+1}-x_{\omega_{1}+1}\right)$.

By definition 28. $\left|x_{0}-x_{\omega_{1}+1}\right| \leq \delta_{\pi}$ and $\left|\left(x_{0}+\eta\right)-x_{\omega_{2}+1}\right| \leq \delta_{\pi}$. Consequently

$$
\eta-2 \delta_{\pi} \leq x_{\omega_{2}+1}-x_{\omega_{1}+1} \leq \eta+2 \delta_{\pi},
$$

which implies that $\frac{x_{\omega_{2}+1}-x_{\omega_{1}+1}}{\eta} \approx 1$. Therefore, $\left(F_{\pi}\left(x_{0}+\eta\right)-F_{\pi}\left(x_{0}\right)\right) / \eta \lesssim M$. Combining with the similar result for $m$, we obtain

$$
m \lesssim \frac{F_{\pi}\left(x_{0}+\eta\right)-F_{\pi}\left(x_{0}\right)}{\eta} \lesssim M
$$

Assuming that $M=f\left(t_{j_{1}}\right)$ and $m=f\left(t_{j_{2}}\right)$, we have $m \approx f\left(x_{0}\right) \approx M$ thanks to continuity and $t_{j_{1}}^{\prime} \approx t_{j_{2}}^{\prime} \approx x_{0}$. Hence, $\Delta_{\eta} F_{\pi}\left(x_{0}\right) \approx f\left(x_{0}\right)$.

The previous theorem can be formulated much more elegantly if we see $\int_{a}^{b} f(x) d x$ and $F^{\prime}(x)$ as ERNA objects 'defined up to infinitesimals' (compare [1, §5]). Accordingly, we interpret an informal statement about $\int_{a}^{b} f(x) d x$ as a statement about all the Riemann sums corresponding to infinitely fine partitions of $[a, b]$. As we can quantify over all partitions of an interval, this informal statement can be expressed in the language of ERNA and we will sometimes forget the distinction between informal and formal terminology. With these conventions, the previous theorem becomes.

30. Corollary (First fundamental theorem of Calculus). Let $f$ be continuous on $[a, b]$ and $F(x)=\int_{a}^{x} f(t) d t$. Then $F$ is S-differentiable on $[a, b]$ and $F^{\prime}(x) \approx f(x)$ holds for all $a \ll x \ll b$.

On a philosophical note, we mention that it seems impossible to develop basic analysis in ERNA (or in any system without a standard-part function) in a quantifier-free way. Indeed, to study the function $F(x)$, we cannot use the quantifier-free definition of differentiability, but we have to revert back to the (standard) non-quantifier-free definition. The same holds for Peano's existence theorem in ERNA, but equally for nonstandard set theory, e.g. the treatment of the nonstandard representative $\frac{\varepsilon}{\varepsilon^{2}+x^{2}}(\varepsilon \approx 0)$ of the Dirac delta function. In 19, we suggest possible solutions for this problem.

Although the proof of theorem 18 may seem straightforward, the condition $\delta_{\pi} / \eta \approx 0$ is highly non-constructive (see also $(18)$ ) and cannot be 'read off' from the first fundamental theorem of calculus. Thus, it seems only fair to say that this theorem, at the very least, does not agree with the spirit of finitism. However, the conditions of the first fundamental theorem can be weakened to remove this problem. Indeed, if $\delta=\frac{\omega_{1}}{\omega_{2}}$, then $\varepsilon^{\prime}=\frac{\left\lfloor\sqrt{\omega_{1}}\right\rfloor}{\left\lceil\sqrt{\omega_{2}}\right\rceil}$ is such that $\delta / \varepsilon^{\prime} \approx 0$. The functions $\lfloor\sqrt{x}\rfloor$ and $\lceil\sqrt{x}\rceil$ are given by the explicit ERNA-formulas

$$
(\mu n \leq x)\left(n^{2} \geq x\right) \text { and }(\mu \leq x)\left(n^{2}<x\right) .
$$

However, there are many more of these conditions and none of them is optimal. 
3.1.5. Differential equations. In this paragraph we prove ERNA's version of the Peano existence theorem for ordinary differential equations. In [24], Sommer and Suppes sketch a proof of this theorem inside ERNA. Their sketch is based on the classical stepwise construction of the function $\phi(x)$ which, in the limit, satisfies the necessary properties. This construction is a prime example of the elegance of nonstandard analysis (see [10]) and we will carry out this construction explicitly inside ERNA in the proof of the following theorem.

31. Theorem (Peano existence theorem). Let $f(x, y)$ be continuous on the rectangle $|x| \leq a,|y| \leq b$, let $M$ be a finite upper bound for $|f|$ there and let $\alpha=\min (a, b / M)$. Then there is a function $\phi$, S-differentiable for $|x| \leq \alpha$, such that

$$
\phi(0)=0 \text { and } \phi^{\prime}(x) \approx f(x, \phi(x)) .
$$

Proof. Without loss of generality, we may assume $a=b=\alpha=1$. We will only consider positive $x$, the proof for negative $x$ is analogous.

First, define $x_{k}:=k / \omega=k \varepsilon$ for $k \leq \omega$ and

$$
y_{m}:=\sum_{k=1}^{m} f\left(x_{k-1}, y_{k-1}\right) \varepsilon \text { and } \phi(x):=\sum_{m=1}^{\omega} T_{\psi}(m, x) y_{m},
$$

where $\psi(m, x) \equiv\left(x_{m-1}<x \leq x_{m}\right)$ and $y_{0}=0$. We verify that $\phi(x)$ satisfies the conditions of the theorem. Fix $0 \ll x \ll 1$ and a nonzero positive infinitesimal $\eta$ such that $\varepsilon / \eta \approx 0$. The case for negative $\eta$ is treated similarly. Now assume that

$$
x_{\omega_{1}-1}<x+\eta \leq x_{\omega_{1}} \text { and } x_{\omega_{2}-1}<x \leq x_{\omega_{2}}
$$

for certain numbers $\omega_{2} \leq \omega_{1} \leq \omega$. Then $\phi(x+\eta)=y_{\omega_{1}}$ and $\phi(x)=y_{\omega_{2}}$ and

$$
\begin{aligned}
\phi(x+\eta)-\phi(x)=y_{\omega_{1}}-y_{\omega_{2}} & =\sum_{k=1}^{\omega_{1}} f\left(x_{k-1}, y_{k-1}\right) \varepsilon-\sum_{k=1}^{\omega_{2}} f\left(x_{k-1}, y_{k-1}\right) \varepsilon \\
& =\varepsilon \sum_{k=\omega_{2}+1}^{\omega_{1}} f\left(x_{k-1}, y_{k-1}\right)
\end{aligned}
$$

Assume $f\left(x_{N}, y_{N}\right)\left(f\left(x_{M}, y_{M}\right)\right.$, respectively) is the largest (the least, respectively) of all $f\left(x_{i}, y_{i}\right)$ for $i$ between $\omega_{1}$ and $\omega_{2}+1$. Define $M^{\prime}=\omega_{1}-\omega_{2}-1$; there holds

$$
\varepsilon M^{\prime} f\left(x_{M}, y_{M}\right) \leq \phi(x+\eta)-\phi(x) \leq \varepsilon M^{\prime} f\left(x_{N}, y_{N}\right)
$$

and also

$$
\frac{\varepsilon}{\eta} M^{\prime} f\left(x_{M}, y_{M}\right) \leq \Delta_{\eta} \phi(x) \leq \frac{\varepsilon}{\eta} M^{\prime} f\left(x_{N}, y_{N}\right) .
$$

By the definition of $x_{n}$, there holds

$$
x_{\omega_{1}-1}-x_{\omega_{2}}=\frac{\omega_{1}-1}{\omega}-\frac{\omega_{2}}{\omega}=\frac{\omega_{1}-\omega_{2}-1}{\omega}=\varepsilon M^{\prime} .
$$

But 23] implies $x_{\omega_{1}-1}-x_{\omega_{2}}<\eta$, which yields $\frac{\varepsilon}{\eta} M^{\prime}<1$. Again by the definition of $x_{n}$, there holds

$$
x_{\omega_{1}}-x_{\omega_{2}-1}=\frac{\omega_{1}}{\omega}-\frac{\omega_{2}-1}{\omega}=\frac{\omega_{1}-\omega_{2}+1}{\omega}=\varepsilon M^{\prime}+2 \varepsilon .
$$

But 23) also implies $x_{\omega_{1}}-x_{\omega_{2}-1}>\eta$, which yields $\frac{\varepsilon}{\eta} M^{\prime}>1-2 \varepsilon / \eta$. Together with $\frac{\varepsilon}{\eta} M^{\prime}<1$, proved above, this yields $\frac{\varepsilon}{\eta} M^{\prime} \approx 1$. It is clear that $x \approx x_{N} \approx x_{M}$. We now prove that $y_{M} \approx y_{N} \approx \phi(x)$. Then 24 and the continuity of $f$ imply

$$
\Delta_{\eta} \phi(x) \approx f\left(x_{N}, y_{N}\right) \approx f\left(x_{M}, y_{M}\right) \approx f(x, \phi(x))
$$

and we are done. 
Assume that $N<M$; the case $N>M$ is treated analogously. From 22 , there follows

$$
y_{N}-y_{M}=\sum_{k=1}^{N} f\left(x_{k-1}, y_{k-1}\right) \varepsilon-\sum_{k=1}^{M} f\left(x_{k-1}, y_{k-1}\right) \varepsilon=\varepsilon \sum_{k=N+1}^{M} f\left(x_{k-1}, y_{k-1}\right) .
$$

By the Weierstrass extremum theorem, $f$ is bounded on $[0,1]$, say by $M^{\prime \prime} \in \mathbb{N}$. Then (22) implies

$$
y_{N}-y_{M} \leq \varepsilon(M-N-1) M^{\prime \prime} \leq \varepsilon\left(\omega_{1}-\omega_{2}-1\right) M^{\prime \prime}=\varepsilon M^{\prime} M^{\prime \prime} .
$$

By the previous, this implies $y_{M} \approx y_{N}$. In the same way, $y_{N} \approx y_{\omega_{2}}=\phi(x)$.

In 24], Sommer and Suppes claim that $\phi(x)$, as defined in (22), is differentiable (in the sense of definition 20. However, due to the absence of a 'standard-part function', the function $\phi(x)$ defined in 22 remains piecewise constant, be it on the infinitesimal level. Thus, if $\eta$ is too small, we have $\phi\left(x_{0}\right)=\phi\left(x_{0}+\eta\right)$ for some $x_{0}$ and hence $\Delta_{\eta} \phi\left(x_{0}\right)=0$, even if $\phi(x)$ is strictly increasing. Hence, it is obvious that $\phi(x)$ cannot be differentiable, but only S-differentiable. Thus, the Peano existence theorem implicitly involves a condition $\varepsilon / \eta \approx 0$ similar to the condition $\delta / \eta \approx 0$ in the fundamental theorem of calculus. As in the latter, S-differentiability hides this technical requirement, but this does not change the fact that $\varepsilon$ - $\delta$-like formulas occur.

Before we continue, we point out that the theorems proved so far fall in either of two fundamentally different classes. A good representative of the first kind is Weierstrass's extremum theorem: it fails when we limit the weight of $x$ and $y$ to $\omega$ in (14). Also, the consequent of this theorem is a property of all numbers in $[a, b]$ of arbitrary depth. On the other hand, the Brouwer fixed point theorem does go through with the aforementioned limitation and its consequent only asserts the existence of a number $x_{0}$ of a certain depth. However, if we were to require a fixed point of arbitrary depth, the resulting 'uniform' fixed point theorem becomes part of the first class. The distinction made here will turn out to be essential in the section 'ERNA and Reverse Mathematics'.

3.2. Mathematics with Transfer. In this section, we prove some well-known results from ordinary mathematics in ERNA $+\Pi_{1}$-TRANS.

First, we prove an ERNA-version of Cauchy completeness, to be understood 'up to infinitesimals'. Indeed, Cauchy completeness is well-known to be equivalent to $\mathrm{ACA}$ over $\mathrm{RCA}_{0}$ and $\mathrm{ACA}_{0}$ has the first-order strength of PA.

32. Definition. A sequence $\tau(n)$ is 'Cauchy' if

$$
\left(\forall^{s t} k\right)\left(\exists^{s t} N\right)\left(\forall^{s t} n\right)\left(\forall^{s t} m\right)\left(n, m \geq N \rightarrow|\tau(n)-\tau(m)|<\frac{1}{k+1}\right)
$$

If $a$ is a constant, we say that a sequence $\tau(n)$ is 'convergent to $a$ ' if

$$
\left(\forall^{s t} k\right)\left(\exists^{s t} N\right)\left(\forall^{s t} n\right)\left(n \geq N \rightarrow|\tau(n)-a|<\frac{1}{k+1}\right)
$$

Clearly, the constant $a$ is only unique up to infinitesimals. We have the following theorem, provable in ERNA $+\Pi_{1}$-TRANS.

33. Theorem (Cauchy completeness). Let $\tau(n)$ be a near-standard Cauchy sequence. Then all $\tau(m)$ are infinitely close to each other for all infinite $m$ and $\tau(n)$ is standard convergent to any of these. 
Proof. If $\tau(n)$ is as required, 28 holds. In this formula, fix any $k \in \mathbb{N}$ and find $N \in \mathbb{N}$ such that

$$
\left(\forall^{s t} n, m\right)\left(n, m \geq N \rightarrow|\tau(n)-\tau(m)|<\frac{1}{k+1}\right) .
$$

In ERNA $+\Pi_{1}$-TRANS, this implies

$$
(\forall n, m)\left(n, m \geq N \rightarrow|\tau(n)-\tau(m)| \lesssim \frac{1}{k+1}\right),
$$

which shows that $\tau(n)$ is convergent to $\tau(m)$ for any infinite $m$. Since 29 can be derived for all $k \in \mathbb{N}$, we have $|\tau(n)-\tau(m)| \lesssim \frac{1}{k+1}$ for all infinite hypernaturals $n, m$ and $k \in \mathbb{N}$. Hence, infinitely indexed terms differ by infinitesimals.

Next, we introduce the well-known $\varepsilon-\delta$ definition of (uniform) continuity in ERNA. This will have immediate consequences for the continuity, differentiability and integration results obtained earlier.

34. Definition. A function $f(x)$ is called ' $S$-continuous over $[a, b]$ ' if

$$
\left(\forall^{s t} k\right)\left(\exists^{s t} N\right)\left(\forall^{s t} x, y \in[a, b]\right)(|x-y|<1 / N \rightarrow|f(x)-f(y)|<1 / k)
$$

35. Theorem. In ERNA, continuity, i.e. (14), implies S-continuity, i.e. (30), for any internal $f(x)$.

Proof. Assume that (14) holds for an internal function $f(x)$. Fix $k \in \mathbb{N}$ and consider the following internal formula $\Phi(n)$

$$
(\forall x, y)((\|x, y\| \leq \omega \wedge|x-y|<1 / n) \rightarrow|f(x)-f(y)|<1 / k) .
$$

By corollary [12, Corollary 53], the above formula is equivalent to a quantifier-free one. By assumption, $\Phi(n)$ holds for all infinite $n$. Hence, by underflow, there is an $N \in \mathbb{N}$ such that $(\forall n \geq N) \Phi(n)$. From this, 30 follows immediately.

36. Theorem. In ERNA $+\Pi_{1}$-TRANS, 30 implies 14 for near-standard functions.

Proof. Let $f(x)$ be a near-standard function which is S-continuous. Fix $1<k \in \mathbb{N}$ and let $N$ be such that

$$
\left(\forall^{s t} x, y \in[a, b]\right)(|x-y|<1 / N \rightarrow|f(x)-f(y)|<1 / k) .
$$

By bar transfer, we obtain

$$
(\forall x, y \in[a, b])(|x-y|<1 / N \rightarrow|f(x)-f(y)| \lesssim 1 / k) .
$$

In particular, $|f(x)-f(y)| \lesssim 1 / k$ if $x \approx y$. But $k \in \mathbb{N}$ can be chosen arbitrarily large and hence $f(x) \approx f(y)$ if $x \approx y$.

The previous theorem has the interesting consequence that all the theorems we obtained in the section 'Mathematics without Transfer' now follow for 'continuous' replaced with 'S-continuous' and 'internal' replaced with 'near-standard'. Thus, we know that $\Pi_{1}$-transfer is sufficient to prove these theorems. In section 4, we show that this transfer principle is exactly what is needed to prove many of these theorems, i.e. $\Pi_{1}$-transfer is also necessary.

For completeness, we note that in ERNA the formula (31) implies 14 for $x$ and $y$ of weight at most some infinite $\omega_{1}$. This is easily proved via overflow in the same way as in 12, Theorem 63]. Thus, ERNA proves the Intermediate value theorem and the Brouwer fixed point theorem.

Next, we prove ERNA's version of $\Sigma_{1}$-separation (see [21, I.11.7 and IV.4.4]). Although ERNA does not have set variables, we can simulate subsets of $\mathbb{N}$ in the following way. Let $(x)_{y}$ be the function which calculates the power of the $(y+1)$-th prime number in the prime decomposition of $x$. It is an easy verification that this 
function is available in ERNA. Thus, we write ' $m \in M$ ' if $(M)_{m}>0$ and in this way, subsets of $\mathbb{N}$ can be mimicked in ERNA (compare [16]).

37. Theorem ( $\Sigma_{1}^{s t}$-separation). For $i=1,2$, let $\psi_{i}$ be formulas $\left(\exists^{s t} m\right) \varphi_{i}(m, n)$ with $\varphi_{i} \in L^{\text {st }}$ quantifier-free. If $\left(\forall^{s t} n\right)\left(\psi_{1}(n) \vee \neg \psi_{2}(n)\right)$, then

$$
(\exists M)\left(\forall^{s t} n\right)\left[\psi_{1}(n) \rightarrow n \in M \wedge \psi_{2}(n) \rightarrow n \notin M\right] .
$$

Proof. Let $\varphi_{i}$ and $\psi_{i}$ be as stated. Define $T(n)$ as true if $(\exists m \leq \omega) \varphi_{1}(m, n)$ and false otherwise. By [12, Corollary 52], the formula $(\exists m \leq \omega) \varphi(m, n)$ is equivalent to a quantifier-free one. By 12 , Theorem 50], the function $T(n)$ is well-defined. It is an easy verification that the function ' $p_{k}=$ the $k$-th prime number' is available in ERNA. Now define the number $M:=\prod_{n=1}^{\omega} p_{n}^{T(n-1)}$. Using $\Pi_{1}$-transfer, it is an easy verification that $M$ has the right properties.

Finally, we prove an upgraded version of the isomorphism theorem (see 23 . Section 6]) in ERNA $+\Pi_{1}$-TRANS. This theorem states that for a finite set of atomic internal propositions in ERNA's language, we can replace each occurrence of ' $\omega$ ', ' $\varepsilon$ ' and ' $x \approx y$ ' with, respectively, ' $n_{0}$ ', ' $1 / n_{0}$ ' and ' $|x-y|<1 / b$ ' (for some $n_{0}, b \in \mathbb{N}$ ) in such a way that the propositions remain true. We first prove the isomorphism theorem and then discuss its philosophical implications. We also discuss why definition 38 is natural in this context. The function $\log ^{k} n$ is defined as $(\mu m \leq n)\left(2_{k}^{m}>n\right)$, where $2_{0}^{m}=m$ and $2_{k+1}^{m}=2^{2_{k}^{m}}$.

38. Definition. An ERNA-term $\tau(\vec{x})$ is called 'intensional' if there is a $k \in \mathbb{N}$ such that ERNA proves $(\forall \vec{x})\left[\|\tau(\vec{x})\|>\log ^{k}(\|\vec{x}\|)\right]$.

Intuitively, a term is intensional if ERNA proves that it grows faster than some iteration of the logarithmic function. By theorem 40, non-intensional terms can have strange behavior and hence they are excluded from the isomorphism theorem, considered next. A closed intensional term is a term of the form $h(\vec{c})$ where $h(\vec{x})$ is intensional and $\vec{c}$ is a tuple of constants.

39. Theorem. Let $\mathcal{T}$ be a finite set of closed intensional terms in the language of ERNA, not including min and closed under subterms. There is a bijection $f$ from $\mathcal{T}$ to a finite set of rationals such that

(i) $f(0)=0, f(1)=1$ and $f(\omega)=n_{0}$, for some $n_{0} \in \mathbb{N}$,

(ii) $f\left(g\left(\tau_{1}, \ldots, \tau_{k}\right)\right)=g\left(f\left(\tau_{1}\right), \ldots, f\left(\tau_{k}\right)\right)$, for all non-atomic terms in $\mathcal{T}$,

(iii) $\tau \approx 0$ iff $|f(\tau)|<\frac{1}{b}$, for some $n_{0}>b \in \mathbb{N}$,

(iv) $\tau$ is infinite iff $|f(\tau)|>b$, for some $n_{0}>b \in \mathbb{N}$,

(v) $\tau$ is hypernatural iff $f(\tau)$ is natural,

(vi) $\sigma \leq \tau$ iff $f(\sigma) \leq f(\tau)$.

Proof. Let $\mathcal{T}$ be as in the theorem and let $D$ be the maximum depth of the terms in $\mathcal{T}$. Complete $\mathcal{T}$ with terms $\lfloor\tau\rfloor$ for $\tau \in \mathcal{T}$, if necessary. By theorem 12 , Theorem 30], there is a $B_{1} \in \mathbb{N}$ such that $\|h(\vec{x})\| \leq 2_{B_{1}}^{\|\vec{x}\|}$ for all terms $h$ in $\mathcal{T}$. As all terms in $\mathcal{T}$ are assumed intensional, there is a $B_{2} \in \mathbb{N}$ such that $\|h(\vec{x})\|>\log ^{B_{2}}(\|\vec{x}\|)$ for all terms $h$ in $\mathcal{T}$. Let $B$ be the maximum of $B_{1}$ and $B_{2}$ and add the term $\log ^{B} \omega$ to $\mathcal{T}$ if necessary.

Then, define $\Psi$ as the conjunction of all true formulas $\mathcal{N}(\tau), \sigma=\tau$ and $\sigma \leq \theta$ with $\tau, \sigma, \theta \in \mathcal{T}$. Let $\Psi(m)$ be $\Psi$ with all occurrences of $\omega$ replaced with the free variable $m$. As $\varepsilon=\frac{1}{\omega}$, any occurrence of $\varepsilon$ in $\Psi$ is replaced with $\frac{1}{m}$. By construction, there holds $\Psi(\omega)$. As $\omega$ is infinite and $2_{2 D B}^{1}$ is finite, this implies $\left(\exists m>2_{2 D B}^{1}\right) \Psi(m)$. By $\Sigma_{1}$-transfer, there holds $\left(\exists^{s t} m>2_{2 D B}^{1}\right) \Psi(m)$, i.e. there is a finite number $m$ such that $m>2_{2 D B}^{1}$ and $\Psi(m)$. Let $m_{0}$ be such a number. Then, let $f$ be any map which maps $\omega$ to $m_{0}$ and has property (ii). By construction, $f$ satisfies (v) 
and vi). To conclude, we show that $f$ also satisfies (iii) and (iv). First of all, by 12. Theorem 30], if $\tau \in \mathcal{T}$ does not involve $\omega$, then it satisfies $\|\tau\| \leq 2_{B D}^{1}$ and hence $\tau$ must be finite. By contraposition, if $\tau \in \mathcal{T}$ is infinite, it must involve $\omega$. Hence we have $\tau=\sigma(\omega)$ for some term $\sigma \in \mathcal{T}$. By assumption, $\lfloor\tau\rfloor$ is also in $\mathcal{T}$ and as all terms in $\mathcal{T}$ are intensional, we have $|\lfloor\sigma(n)\rfloor| \geq \log ^{B} n$. In particular, we have, for $\tau>0$,

$$
f(\lfloor\tau\rfloor)=f(\lfloor\sigma(\omega)\rfloor)=\lfloor\sigma(f(\omega))\rfloor=\left\lfloor\sigma\left(m_{0}\right)\right\rfloor \geq \log ^{B} m_{0} .
$$

Thus, if $\tau>0$ is infinite, then $f(\lfloor\tau\rfloor) \geq \log ^{B} m_{0}$, which implies $f(\tau) \geq \log ^{B} m_{0}$. Hence, for all infinite $\tau \in \mathcal{T}$, we have $|f(\tau)| \geq \log ^{B} m_{0}$. Now assume that $|f(\tau)| \geq$ $\log ^{B} m_{0}$ for some $\tau \in \mathcal{T}$. This yields $|f(\tau)| \geq f\left(\log ^{B} \omega\right)$ and, by item (vi), there holds $|\tau| \geq \log ^{B} \omega$. Thus, $\tau$ is infinite and we have proved item (iv) for $b=\log ^{B} m_{0}$. As item (iv) implies item (iii), we are done.

In comparison to [23, we removed the 'reasonably sound' condition from the isomorphism theorem, which is a significant improvement (compare 23, Theorem 6.1]), and we formulated a correct proof. However, we added the 'intensionality' condition and it may not be clear why this condition is natural. We give several arguments, both heuristic and formal. We work in $I \Delta_{0}+\exp$ because although theorem 40 can be generalised to ERNA $+\Pi_{1}$-TRANS, this is beyond the scope of this paper.

First of all, the best-known example of a non-intensional function is $\log ^{*} n=$ $(\mu k \leq n)\left(\log ^{k} n \leq 1\right)$. It can be computed that for $n_{0}=2^{65536}$, which is larger than the number of particles in the universe, $\log ^{*} n_{0}$ is at most five. Thus, for practical purposes, $\log ^{*} n$ may be regarded as a constant function. The following theorem makes this qualitative statement precise and more convincing.

40. Theorem. The theory $I \Delta_{0}+\exp$ cannot prove that the function $\log ^{*} x$ is unbounded, i.e. $I \Delta_{0}+\exp \forall(\forall x)(\exists y)\left(\log ^{*} y>x\right)$.

Proof. Assume to the contrary that $I \Delta_{0}+\exp$ proves $(\forall x)(\exists y)\left(\log ^{*} y>x\right)$. By Parikh's theorem (see [5. Theorem 1.2.7.1]), there is a term $t$ such that $I \Delta_{0}+\exp$ proves the sentence $(\forall x)(\exists y \leq t(x))\left(\log ^{*} y>x\right)$. As $\log ^{*} x$ is weakly increasing, there follows that $(\forall x)\left(\log ^{*}(t(x))>x\right)$. However, this implies that $t(x)$ grows faster than all $2_{k}^{x}$, which is impossible.

By completeness, there is a model of $I \Delta_{0}+\exp$ in which $\log ^{*} x$ is bounded. From the point of view of logic, this model is 'nonstandard' and 'exotic'. However, given the slow-growing nature of $\log ^{*} x$ discussed above, we perceive this function as constant or bounded above in the 'real world'. Thus, this 'exceptional' model is natural from the anthropocentric point of view. Since the isomorphism theorem is intended to deal with models of physical problems, it seems reasonable to choose a model of ERNA which corresponds to the real world, i.e. one where $\log ^{*} n$ is constant. Another interpretation of theorem 40 is that since $I \Delta_{0}+$ exp cannot even prove that non-intensional terms are unbounded (whereas e.g. PRA can), we might as well exclude such terms from the isomorphism theorem, as we cannot learn anything about them in $I \Delta_{0}+\exp$ anyway.

We now discuss the philosophical implications of the isomorphism theorem.

First of all, it shows that the use of irrational numbers (and functions taking such values) in physics is merely a convenient calculus tool. Indeed, let $\mathcal{M}$ be a model of a (necessarily finite) physical problem $\mathcal{P}$ that involves irrational numbers. We can approximate these numbers by hyperrationals with infinitesimal precision. 
After replacing the irrational numbers with these approximations, we apply the isomorphism theorem to obtain a model $\mathcal{M}^{\prime}$ of $\mathcal{P}$ that only involves rational numbers. We second Sommer and Suppes' claim that 'the continuum may be real for Platonists, but it can nowhere be unequivocally identified in the real world of physical experiments.' (see 23, Introduction]).

Secondly, the representation of physical quantities such as space and time as continuous variables is called into question by the isomorphism theorem. Indeed, by the latter, a discrete set of rational numbers already suffices to model a physical problem and hence no physical experiment can decide the 'true' nature (discrete or continuous) of physical quantities. The obvious way to avoid the previous 'undecidability' result, is to simply state that one does not accept the isomorphism theorem (or $\Pi_{1}$-transfer) and hence one is not bound to its implications. We counter with the following observation: by theorem 2 we know that $\Pi_{1}$-transfer is equivalent to the 'continuity principle' that $\varepsilon-\delta$ continuity implies nonstandard continuity. The latter formalizes the heuristic notion of continuity, which is fundamental in the informal reasoning inherent to applied sciences, especially physics. Thus, the continuity principle is inherent to physics and so is $\Pi_{1}$-transfer. From the latter, the isomorphism theorem follows. To the best of our knowledge, this is the first example of an application of Reverse Mathematics to physics.

\section{Reverse Mathematics And ERNA}

4.1. A copy of Reverse Mathematics for $\mathbf{W K L}_{0}$. In this section, we prove the equivalences between $\Pi_{1}$-transfer and theorems of ordinary mathematics listed in theorem 2. Most of the latter are derived from theorems equivalent to Weak König lemma (see theorem 1 and [21]) by replacing equality with ' $\approx$ '. Hence the Reverse Mathematics for ERNA $+\Pi_{1}$-TRANS is a 'copy' of the Reverse Mathematics for $\mathrm{WKL}_{0}$, up to infinitesimals.

We also mention Strict Reverse Mathematics (SRM), recently introduced by Harvey Friedman, which is 'a form of Reverse Mathematics relying on no coding mechanisms, where every statement considered must be strictly mathematical'. Comparing the usual definition of continuity with [21, Definition II.6.1, p. 85], it is clear that Reverse Mathematics uses significant coding machinery. In contrast, ERNA can approximate most functions that appear in mathematical practice by near-standard functions and bar transfer enables us to prove many well-known results and the associated reversal, all with minimal coding. Thus, the Reverse Mathematics of ERNA $+\Pi_{1}$-TRANS is also a contribution to SRM.

4.2. Completeness. Recall theorem 33 which expresses that ERNA's field is Cauchy complete 'up to infinitesimals'. Thus, in the context of ERNA, we refer to this theorem as the 'Cauchy completeness principle'. We have the following theorem.

41. Theorem. In ERNA, $\Pi_{1}$-transfer is equivalent to the Cauchy completeness principle.

Proof. By theorems 9 and 33 the forward implication is immediate. To obtain the reverse implication, assume the Cauchy completeness principle holds, let $\varphi$ be as in $\Pi_{1}$-TRANS and assume $\varphi(m)$ for $m \in \mathbb{N}$. Let $\tau(n)$ be a near-standard Cauchy sequence and define the sequence

$$
\sigma(n)= \begin{cases}\tau(n) & (\forall m \leq n) \varphi(m) \\ n & \text { otherwise }\end{cases}
$$

By definition 3, $\sigma(n)$ is also near-standard. By assumption $\sigma(n)=\tau(n)$ for $n \in \mathbb{N}$ and hence $\sigma(n)$ is also a Cauchy sequence. By Cauchy completeness, we have $\sigma(k) \approx$ 
$\sigma\left(k^{\prime}\right)$ for all infinite $k, k^{\prime}$. If $\sigma(k)=k$ for some infinite $k$, then also $\sigma(k+1)=k+1$, by definition. But then $\sigma(k) \not \approx \sigma(k+1)$, which yields a contradiction. Thus, for all infinite $k$, there must hold $\sigma(k)=\tau(k)$. By definition, this implies $\varphi(m)$ for all $m$ and hence $\Pi_{1}$-TRANS follows.

4.3. Continuity and integration. Consider the following 'continuity principle'.

42. Principle (Continuity principle). For near-standard functions, S-continuity implies continuity, i.e. (30) implies (14).

43. Theorem. In ERNA, the continuity principle is equivalent to $\Pi_{1}$-TRANS.

Proof. The reverse implication is immediate from theorem 36 . Conversely, assume the continuity principle and consider a quantifier-free formula $\varphi$ of $L^{s t}$, such that $\varphi(n)$ holds for $n \in \mathbb{N}$. Let $f$ be a near-standard S-continuous function on $[a, b]$. By cases, we define the near-standard function

$$
g(x)=\left\{\begin{array}{ll}
f(x) & (\forall n \leq\|x\|) \varphi(n) \\
\|x\| & \text { otherwise }
\end{array} .\right.
$$

For standard $x$, we have $\|x\| \in \mathbb{N}$ and $(\forall n \leq\|x\|) \varphi(n)$ holds by assumption. Hence, for standard $x, g(x)=f(x)$, the latter being a function S-continuous over $[a, b]$. Hence $g(x)$ is S-continuous over $[a, b]$ too and, by assumption, this implies that $g(x)$ is continuous over $[a, b]$. Now suppose there is an infinite $k$ such that $\neg \varphi(k)$ and let $k_{0}$ be the least number with this property. Fix $a \ll x_{0} \ll b$ with weight $\leq k_{0}$. Assume $k_{1} \geq k_{0}$ is prime. By $(13),\left\|x_{0}+1 / k_{1}\right\| \geq k_{1}$ and thus we have $g\left(x_{0}+1 / k_{1}\right)=\left\|x+1 / k_{1}\right\|$, where the latter is infinite. But by assumption $g(x)$ is continuous, which implies $g\left(x_{0}\right) \approx g\left(x_{0}+1 / k_{1}\right)$, as $x_{0} \approx x_{0}+1 / k_{1}$. Since $g\left(x_{0}\right)=$ $f\left(x_{0}\right)$, the latter is a finite number by corollary 14 . This yields a contradiction and hence $\varphi(n)$ must hold for all $n$. This implies $\Pi_{1}$-TRANS

In the previous proof, ERNA's weight function $\|x\|$ is used not as a proof theoretic tool (as in the consistency proof of [23]), but as an ERNA-function that is everywhere discontinuous. However, from the proof of the theorem, it is clear that we could replace it by a function which has a jump in its graph for some $a \ll x_{0} \ll b$. Indeed, in the proof, we only consider continuity in the point $a \ll x_{0} \ll b$. Thus, the theorem still holds if we only require $f$ to be continuous over $(a, b)$ in the continuity principle. We will refer to this as the 'continuity principle' too.

Now consider the following version of Weierstrass' extremum theorem.

44. Principle (Weierstrass extremum principle). If $f$ is near-standard and $S$ continuous over $[a, b]$, there is a number $c \in[a, b]$ such that for all $x \in[a, b]$, we have $|f(x)| \lesssim|f(c)|$.

45. Theorem. In ERNA, the Weierstrass extremum principle is equivalent to $\Pi_{1}$-TRANS.

Proof. The reverse implication is immediate from theorems 12 and 36 . Conversely, assume the Weierstrass extremum principle and consider a quantifier-free formula $\varphi$ of $L^{s t}$ such that $\varphi(n)$ is valid for all $n \in \mathbb{N}$. Define $g(x)$ as in (32). In the same way as in the previous proof, $g$ is S-continuous over $[a, b]$ and by the Weierstrass extremum principle there is a number $c \in[a, b]$ such that $|g(x)| \lesssim|g(c)|$, for all $x \in[a, b]$. Now suppose there is an $n_{0}$ such that $\neg \varphi\left(n_{0}\right)$. By theorem 10 there is an $a \ll x_{0} \ll b$ with weight at least $1+\left\lceil\max \left\{n_{0},|g(c)|\right\}\right\rceil$. As $\left\|x_{0}\right\|>n_{0}$, this implies $\left(\exists n \leq\left\|x_{0}\right\|\right) \neg \varphi(n)$ and by the definition of $g$, we have $\left|g\left(x_{0}\right)\right|=\left\|x_{0}\right\|$. But by the definition of $x_{0}$, there also holds $\left|g\left(x_{0}\right)\right|=\left\|x_{0}\right\| \gg|g(c)|$. This is a contradiction and hence $\varphi(n)$ must hold for all $n$, which implies $\Pi_{1}$-TRANS. 
Note that the proof can be easily adapted to a weaker version of Weierstrass' extremum principle where $|f(x)|$ is only bounded by some $M \in \mathbb{N}$ for $x \in[a, b]$.

Next, we treat Brouwer's fixed point theorem. We need the following definition.

46. Definition. The point $x_{0}$ is a 'fixed point up to infinitesimals' of $f$ if $f\left(x_{0}\right) \approx x_{0}$.

After theorem 36, it was noted before that in ERNA every S-continuous function has a fixed point up to infinitesimals. As $\mathrm{RCA}_{0}$ proves the one-dimensional Brouwer fixed point theorem, this supports our claim concerning the resemblance between the Reverse Mathematics of $\mathrm{WKL}_{0}$ and that of ERNA $+\Pi_{1}$-TRANS. However, the following strengthening of Brouwer fixed point theorem is not provable in ERNA.

47. Principle (Uniform Brouwer fixed point principle). For every function $f$, nearstandard and $S$-continuous over $[a, b]$, there is a fixed point up to infinitesimals of arbitrary weight.

48. Theorem. In ERNA, the Uniform Brouwer fixed point principle is equivalent to $\Pi_{1}$-TRANS.

Proof. The reverse implication, is immediate from theorem 36 and the Brouwer fixed point theorem. Conversely, assume the Uniform Brouwer fixed point principle and consider a quantifier-free formula $\varphi$ of $L^{\text {st }}$ such that $\varphi(n)$ is valid for all $n \in \mathbb{N}$. Define $g(x)$ as in $(32)$. In the same way as in the previous proofs, $g$ is S-continuous over $[a, b]$. Now suppose $\neg \varphi\left(n_{0}\right)$ for some infinite $n_{0}$. By the Uniform Brouwer fixed point principle, there is a point $x_{0} \in[a, b]$ with weight at least $n_{0}$ such that $g\left(x_{0}\right) \approx x_{0}$. If $g\left(x_{0}\right)$ equals $\left\|x_{0}\right\|$, there holds $\left\|x_{0}\right\| \approx x_{0}$, which is obviously false. Thus, we have $g\left(x_{0}\right)=f\left(x_{0}\right)$, which implies $\left(\forall n \leq\left\|x_{0}\right\|\right) \varphi(n)$, by definition. As $\left\|x_{0}\right\| \geq n_{0}$, this yields $\varphi\left(n_{0}\right)$, which contradicts $\neg \varphi\left(n_{0}\right)$. Thus, $\varphi(n)$ holds for all $n$ and we obtain $\Pi_{1}$-TRANS.

Finally, we consider the following principle concerned with Riemann integration.

49. Principle (Riemann integration principle). A near-standard function which is $S$-continuous over $[a, b]$, is integrable there.

50. Theorem. In the theory ERNA, the Riemann integration principle is equivalent to $\Pi_{1}$-TRANS.

Proof. The reverse implication is immediate from theorems 19 and 36. Conversely, assume that the Riemann integration principle holds and consider a quantifier-free formula $\varphi$ of $L^{s t}$ such that $\varphi(n)$ is valid for all $n \in \mathbb{N}$. Let $g(x)$ be as in (32). As $\varphi(n)$ is true for all $n \in \mathbb{N}$, we have $g(x)=f(x)$ for all standard $x$ and hence the Riemann integration principle applies to $g$. Overflow applied to $\left(\forall^{s t} n\right) \varphi(n)$ yields $\left(\forall n \leq \omega_{1}\right) \varphi(n)$ and hence $g(x)=f(x)$ for all $x$ such that $\|x\| \leq \omega_{1}$. Then put $\omega_{2}=\left\lfloor\omega_{1} / 2\right\rfloor$ and consider the equidistant partition with mesh $1 / \omega_{2}$ and points $t_{i}=\frac{x_{i+1}-x_{i}}{2}$. As $\left\|t_{i}\right\| \leq \omega_{1}$, it is clear that $g\left(t_{i}\right)=f\left(t_{i}\right)$ for $1 \leq i \leq \omega_{2}$ and assume the Riemann sum of $f$ corresponding to this partition is the finite number $S$.

Now suppose there is a (necessarily infinite) hypernatural $n_{0}$ such that $\neg \varphi\left(n_{0}\right)$. By the definition of $g(x)$, there follows $g(x)=\|x\|$ if $\|x\| \geq n_{0}$. Then consider the equidistant partition with mesh $1 / n_{0}$ and points $t_{i}=\frac{x_{i+1}-x_{i}}{2}$. The corresponding Riemann sum is easily calculated:

$$
\sum_{i=1}^{n_{0}} g\left(t_{i}\right)\left(x_{i}-x_{i-1}\right)=\sum_{i=1}^{n_{0}}\left\|t_{i}\right\| \frac{1}{n_{0}}=\sum_{i=1}^{n_{0}} 2 n_{0} \frac{1}{n_{0}}=2 n_{0} .
$$

By the Riemann integration principle, there holds $S \approx 2 n_{0}$. Obviously, this is impossible and the assumption that there is a number $n_{0}$ such that $\neg \varphi\left(n_{0}\right)$ is false. This implies $\Pi_{1}$-TRANS and we are done 
Theorem 43 suggests an alternative proof for the forward implication. Indeed, assume the Riemann integration principle and suppose there are $x_{0}, y_{0} \in[a, b]$ such that $x_{0} \approx y_{0}$ and $f\left(x_{0}\right) \not f\left(y_{0}\right)$. Assume $x_{0}<y_{0}$ and fix an infinitely fine partition $\pi$ of $[a, b]$ for which $x_{i}<x_{0}<y_{0}<x_{i+1}$ and $x_{0}=t_{i}$ for some $i$. Change $\pi$ into $\pi^{\prime}$ by putting $y_{0}=t_{i}$. It is easily checked that the Riemann sums corresponding to $\pi$ and $\pi^{\prime}$ differ a finite amount and we have a contradiction. Thus $f$ is continuous on $[a, b]$ and theorem 43 implies $\Pi_{1}$-transfer.

4.4. Integration and differentiability. Consider the following version of the first fundamental theorem of calculus.

51. Principle $\left(\mathrm{FTC}_{1}\right)$. Let $f$ be near-standard and $S$-continuous on $[a, b]$ and assume $F(x)=\int_{a}^{x} f(t) d t$. Then $F$ is S-differentiable on $[a, b]$ and $F^{\prime}(x) \approx f(x)$ holds for all $a \ll x \ll b$.

52. Theorem. In ERNA, $\mathrm{FTC}_{1}$ is equivalent to $\Pi_{1}$-TRANS.

Proof. The reverse implication is immediate by corollary 30 and theorem 36 . For the forward implication, assume $\mathrm{FTC}_{1}$ and let $f$ be as stated there. By $\mathrm{FTC}_{1}, F(x)$ is S-differentiable and hence $F^{\prime}(x)$ is continuous, by corollary 24. Again, by $\mathrm{FTC}_{1}$, the formula $F^{\prime}(x) \approx f(x)$ holds for all $a \ll x \ll b$ and hence $f(x)$ is also continuous over $(a, b)$. By theorem 43 , this implies $\Pi_{1}$-TRANS and we are done.

Consider the following version of the Peano existence theorem.

53. Principle (Peano existence principle). Let $f(x, y)$ be near-standard and $S$ continuous on the rectangle $|x| \leq a,|y| \leq b$, let $M$ be a finite upper bound for $f$ there and let $\alpha=\min (a, b / M)$. Then there is a function $\phi$, S-differentiable for $|x|<\alpha$, such that

$$
\phi(0)=0 \text { and } \phi^{\prime}(x) \approx f(x, \phi(x)) .
$$

54. Theorem. In ERNA, the Peano existence principle is equivalent to $\Pi_{1}$-TRANS.

Proof. The reverse implication is immediate by theorem 31 and theorem 36 . For the forward implication, we prove that the function $\phi^{\prime}(x)$ is continuous in the same way as for $\mathrm{FTC}_{1}$. Then it is immediate that $f(x, \phi(x))$ is continuous over $(a, b)$. From this, $\Pi_{1}$-TRANS follows in the same way as in the proof of theorem 43 .

4.5. Approximation and Bernstein polynomials. In this paragraph, we study an ERNA-version of the Weierstrass approximation theorem. The latter is equivalent to WKL over $\mathrm{RCA}_{0}$ (see 21, Theorem IV.2.5]).

55. Definition. For a function $f$, define the $n$-th Bernstein polynomial as

$$
B_{n}(f)(x):=\sum_{k=0}^{n} f(k / n)\left(\begin{array}{l}
n \\
k
\end{array}\right) x^{k}(1-x)^{n-k} .
$$

56. Principle (Weierstrass approximation principle). Let $f$ be near-standard and $S$-cont. on $[a, b]$. Then $B_{m}(f)(x) \approx f(x)$ for all $x \in[a, b]$ and infinite $m$.

57. Theorem. In ERNA, the Weierstrass approximation theorem is equivalent to $\Pi_{1}$-TRANS.

Proof. Assume $\Pi_{1}$-TRANS. It suffices to prove the Weierstrass approximation principle for $[a, b]$ equal to $[0,1]$. In $[7]$, an elementary, rather tedious, proof of the Weierstrass approximation theorem is given, based on Bernstein's original proof. This proof can easily be adapted to the context of ERNA to prove

$$
\left(\forall^{s t} k\right)\left(\exists^{s t} N\right)\left(\forall^{s t} n \geq N\right)\left(\forall^{s t} x \in[0,1]\right)\left(\left|B_{n}(f)(x)-f(x)\right|<1 / k\right) .
$$


Applying transfer to the innermost universal formula implies $B_{m}(f)(x) \approx f(x)$ for all $x \in[0,1]$ and all infinite $m$.

Now assume the Weierstrass approximation theorem and let $f$ be as stated there. Its a technical verification that ERNA proves that $B_{m}(f)(x)$ is continuous on $[a, b]$ for small enough infinite $m$. Since $B_{m}(f)(x) \approx f(x)$ for all $x \in[a, b]$ and infinite $m$, this implies the continuity of $f$ on $[a, b]$ and theorem 43 yields $\Pi_{1}$-TRANS.

4.6. Modulus of uniform continuity. In this paragraph, we study ERNA's version of the 'modulus of uniform continuity' (see [21, Definition IV.2.1]). The statement 'every uniform continuous function has a modulus of uniform continuity' is equivalent to WKL over $\mathrm{RCA}_{0}([21, \mathrm{IV} .2 .9])$. We use the following weaker definition. Indeed, our modulus depends on $\|x, y\|$.

58. Definition. Let $f$ be a function defined on $[a, b]$. A function $h(k, m)$ is a modulus of uniform continuity for $f$ if for all $m$ we have

$$
\begin{aligned}
\left(\forall^{s t} k\right)(\forall x, y \in[a, b])[\|x, y\| & \leq m \\
& \left.\wedge|x-y|<\frac{1}{h(k, m)} \rightarrow|f(x)-f(y)|<\frac{1}{k+1}\right],
\end{aligned}
$$

and $h(k, m)$ is finite for finite $k$.

59. Principle (Modulus principle). Every near-standard function, S-continuous on $[a, b]$, has a modulus of uniform continuity.

60. Theorem. In ERNA, the modulus principle is equivalent to $\Pi_{1}$-TRANS.

Proof. First, assume $\Pi_{1}$-TRANS and let $f$ be as in the modulus principle. Then,

$$
\left(\forall^{s t} k\right)\left(\exists^{s t} N\right)\left(\forall^{s t} x, y \in[a, b]\right)\left[|x-y|<\frac{1}{N} \rightarrow|f(x)-f(y)|<\frac{1}{k+2}\right],
$$

and by bar transfer

$$
\left(\forall^{s t} k\right)\left(\exists^{s t} N\right)(\forall x, y \in[a, b])\left[|x-y|<\frac{1}{N} \rightarrow|f(x)-f(y)| \lesssim \frac{1}{k+2}\right],
$$

and also

$$
\left(\forall^{s t} k\right)\left(\exists^{s t} N\right)(\forall x, y \in[a, b])\left[|x-y|<\frac{1}{N} \rightarrow|f(x)-f(y)|<\frac{1}{k+1}\right] .
$$

Thus, for any fixed $m$, there holds

$$
\left(\forall^{s t} k\right)\left(\exists^{s t} N\right)(\forall x, y \in[a, b])\left[\|x, y\| \leq m \wedge|x-y|<\frac{1}{N} \rightarrow|f(x)-f(y)|<\frac{1}{k+1}\right] .
$$

By [12, Corollary 53], the innermost universal formula is equivalent to quantifierfree formula. Then define $h(k, m)$ as

$$
(\mu N \leq \omega)(\forall x, y \in[a, b])\left[\|x, y\| \leq m \wedge|x-y|<\frac{1}{N} \rightarrow|f(x)-f(y)|<\frac{1}{k+1}\right],
$$

which is a suitable modulus.

For the forward implication, assume the modulus principle and let $f$ be as stated there. Then $f$ satisfies $(33)$ for some modulus $h(k, m)$. Now fix $x_{0}, y_{0} \in[a, b]$ such that $x_{0} \approx y_{0}$ and apply (33) for $m_{0}=\left\|x_{0}, y_{0}\right\|$. This implies that $f\left(x_{0}\right) \approx f\left(y_{0}\right)$ and hence $f$ is also continuous over $[a, b]$. By theorem $43, \Pi_{1}$-TRANS follows and we are done. 
4.7. Conclusion. We concluded the proof of theorem 2 and we repeat our dictum.

The Reverse Mathematics of ERNA $+\Pi_{1}$-TRANS is a 'copy up to infinitesimals' of the Reverse Mathematics of $\mathrm{WKL}_{0}$.

However, one slight anomaly is present: the Cauchy completeness property is equivalent to ACA over $\mathrm{RCA}_{0}$ ([21, Theorem III.2.2]), but ERNA's version of Cauchy completeness is equivalent to $\Pi_{1}$-TRANS. In section 5 , we give a possible explanation for this phenomenon.

Finally, we note that the list in theorem 2 is not exhaustive. We point to 26 where Keita Yokoyama proves the equivalence between WKL and Cauchy's integral theorem which states that a complex function $f \in C^{1}(\Omega)$ satisfies the well-known zero-law $\oint_{\gamma} f(z) d z=0$ for a sufficiently well-behaved closed curve $\gamma \subset \Omega$. It is beyond the scope of this paper to develop complex analysis in ERNA, but we mention that $\Pi_{1}$-TRANS is equivalent to an ERNA-version of the Cauchy integral theorem with 'approximate' zero-law $\oint_{\gamma} f(z) d z \approx 0$.

Similarly the Jordan curve theorem is equivalent to $\mathrm{WKL}_{0}([18])$ and ERNA's version of this theorem would only imply that for every arc $A(x)$ with endpoints in the interior and exterior of the Jordan curve $J(x)$, there is a point $x_{0}$ such that $A\left(x_{0}\right) \approx J\left(x_{0}\right)$. Thus, the Jordan curve and the arc only meet 'up to infinitesimals', consistent with our dictum.

\section{ERNA and Constructive Reverse Mathematics}

In this section we speculate on the connection between the Reverse Mathematics for ERNA $+\Pi_{1}$-TRANS and Constructive Reverse Mathematics. We first briefly introduce the latter.

Constructive mathematics ( $2 \sqrt{4})$ is described by Douglas Bridges as 'that mathematics which is characterised by numerical content and computational method.' ([3, p. 1]). Thus, in constructive mathematics, the quantifier ' $(\exists x)$ ' means 'there is an algorithm to compute the object $x$ '. This is stronger than the 'ideal' notion of existence in the sense of Plato used in classical mathematics. From the constructive perspective, the law of excluded is suspect since it carries non-constructive content and therefore it is excluded from constructive mathematics. Constructive Reverse Mathematics studies equivalences between both constructive and nonconstructive theorems in a constructive base theory (see e.g. 14 15]). In the case of non-constructive theorems, one of the goals is to find out just how much of the law of excluded middle (or another non-constructive principle) is needed to prove such a theorem. In this context, the following principle occurs in relation to Cauchy completeness.

61. Principle $\left(\Sigma_{1}\right.$-PEM). For all quantifier-free $\varphi$, there holds

$$
(\exists n) \varphi(n) \vee(\forall n) \neg \varphi(n) .
$$

In the previous, the existential quantifier ' $(\exists n)$ ' means that 'a number $n$ can be computed'. Also, $\Pi_{1}$-transfer is equivalent to the following schema.

62. Principle $\left(\Sigma_{1}\right.$-TRANS). For all quantifier-free $\varphi \in L^{s t}$, there holds

$$
\left(\exists^{s t} n\right) \varphi(n) \vee(\forall n) \neg \varphi(n) .
$$

In this way, $\Sigma_{1}$-TRANS is a form of 'hyperexcluded middle': it excludes the possibility that $\left(\forall^{s t} n\right) \varphi(n) \wedge(\exists n) \neg \varphi(n)$. Not only does $\Sigma_{1}$-transfer resemble $\Sigma_{1^{-}}$ PEM, we can also easily compute a witness to $\left(\exists^{s t} n\right) \varphi(n)$ by the number $(\mu n \leq$ $\omega) \varphi(n)$. Thus, we see that $\Pi_{1}$-transfer has significant constructive content, similar 
to that of $\Sigma_{1}$-PEM. As the latter is related to Cauchy completeness, it is no surprise that $\Pi_{1}$-transfer is also related to Cauchy completeness (see theorem 2, (8)).

We can take this analogy further by considering another principle from Constructive Reverse Mathematics related to Cauchy completeness.

63. Principle $\left(\Pi_{1}^{0}-\mathrm{AC}_{00}\right)$. For $A \in \Pi_{1}$, we have

$$
(\forall m)(\exists n) A(m, n) \rightarrow(\exists \alpha)(\forall m) A(m, \alpha(m)) .
$$

In constructive mathematics, this choice principle implies that every Cauchy sequence has a modulus. In ERNA $+\Pi_{1}$-TRANS, we have the following theorem.

64. Theorem (Countable Universal Choice). Assume $A(m, n)$ is $\left(\forall^{s t} k\right) B(k, m, n)$ with $B \in L^{\text {st }}$ and quantifier-free. Then $\left(\forall^{s t} m\right)\left(\exists^{s t} n\right) A(m, n)$ implies the formula $\left(\forall^{s t} m\right) A(m, \alpha(m))$ for some nonstandard function $\alpha$.

Proof. Let $A(m, n)$ be as stated. By transfer, $\left(\forall^{s t} m\right)\left(\exists^{s t} n\right) A(m, n)$ implies the formula $\left(\forall^{s t} m\right)\left(\exists^{s t} n\right)(\forall k) B(k, m, n)$. This yields $\left(\forall^{s t} m\right)\left(\exists^{s t} n\right)(\forall k \leq \omega) B(k, m, n)$ and the function $\alpha(m)=(\mu n \leq \omega)(\forall k \leq \omega) B(k, m, n)$ is a suitable modulus.

The previous theorem can be modified to be equivalent to $\Pi_{1}$-TRANS.

65. Acknowledgement. I thank Professor Andreas Weiermann (University of Ghent) and Professor Chris Impens (University of Ghent) for their valuable advice.

\section{REFERENCES}

[1] Jeremy Avigad, Weak theories of nonstandard arithmetic and analysis, Reverse mathematics 2001, Lect. Notes Log., vol. 21, Assoc. Symbol. Logic, La Jolla, CA, 2005, pp. 19-46.

[2] Errett Bishop and Douglas S. Bridges, Constructive analysis, Grundlehren der Mathematischen Wissenschaften [Fundamental Principles of Mathematical Sciences], vol. 279, SpringerVerlag, Berlin, 1985. MR804042 (87d:03172)

[3] Douglas S. Bridges, Constructive functional analysis, Research Notes in Mathematics, vol. 28, Pitman publishing, London, San Francisco and Melbourne, 1979.

[4] Douglas S. Bridges and Luminiţa Simona Vîţă, Techniques of constructive analysis, Universitext, Springer, New York, 2006. MR2253074 (2008a:03109)

[5] Samuel R. Buss, An introduction to proof theory, Handbook of proof theory, Stud. Logic Found. Math., vol. 137, North-Holland, Amsterdam, 1998, pp. 1-78.

[6] Rolando Chuaqui and Patrick Suppes, Free-variable Axiomatic Foundations of Infinitesimal Analysis: A Fragment with Finitary Consistency Proof, Journal of Symbolic Logic 60 (1995), $122-159$.

[7] Kenneth R. Davidson and Allan P. Donsig, Real Analysis with Real Applications, Prentice Hall, Upper Saddle River, N.J., 2002.

[8] Harvey Friedman, Some systems of second order arithmetic and their use, Proceedings of the International Congress of Mathematicians (Vancouver, B. C., 1974), Vol. 1, Canad. Math. Congress, Montreal, Que., 1975, pp. 235-242. MR0429508 (55 \#2521)

[9] _ Systems of second order arithmetic with restricted induction, I \& II (Abstracts), Journal of Symbolic Logic 41 (1976), 557-559.

[10] Albert E. Hurd and Peter A. Loeb, An introduction to nonstandard real analysis, Pure and Applied Mathematics, vol. 118, Academic Press Inc., Orlando, FL, 1985.

[11] Chris Impens and Sam Sanders, ERNA at work, The strength of nonstandard analysis, Springer Wien NewYork Vienna, 2007, pp. 64-75.

[12] 689-710.

[13] S Saturation and $\Sigma_{2}$-transfer for ERNA, Journal of Symbolic Logic 74 (2009), 901-913.

[14] Hajime Ishihara, Reverse mathematics in Bishop's constructive mathematics, Philosophia Scientiae (Cahier Spécial) 6 (2006), 43-59.

[15] _ Constructive reverse mathematics: compactness properties, From sets and types to topology and analysis, Oxford Logic Guides, vol. 48, Oxford Univ. Press, Oxford, 2005, pp. 245-267. MR2188648 (2006f:03113)

[16] H. Jerome Keisler, Nonstandard arithmetic and reverse mathematics, Bull. Symbolic Logic 12 (2006), no. 1, 100-125. MR2209331 (2006m:03092) 
[17] Michal Rössler and Emil Jeřábek, Fragment of nonstandard analysis with a finitary consistency proof, Bulletin of Symbolic Logic 13 (2007), 54-70.

[18] Nobuyuki Sakamoto and Keita Yokoyama, The Jordan curve theorem and the Schönflies theorem in weak second-order arithmetic, Arch. Math. Logic 46 (2007), no. 5-6, 465-480.

[19] Sam Sanders, More infinity for a better finitism, Submitted (2009).

[20] Stephen G. Simpson, Which set existence axioms are needed to prove the Cauchy/Peano theorem for ordinary differential equations?, J. Symbolic Logic 49 (1984), no. 3, 783-802.

[21] _ Subsystems of second order arithmetic, 2nd ed., Perspectives in Logic, Cambridge University Press, Cambridge, 2009. MR2517689

[22] (ed.), Reverse mathematics 2001, Lecture Notes in Logic, vol. 21, Association for Symbolic Logic, La Jolla, CA, 2005. MR2186912 (2006f:03003)

[23] Richard Sommer and Patrick Suppes, Finite Models of Elementary Recursive Nonstandard Analysis, Notas de la Sociedad Mathematica de Chile 15 (1996), 73-95.

[24] _ Dispensing with the Continuum, Journal of Math. Psychology 41 (1997), 3-10.

[25] Patrick Suppes and Rolando Chuaqui, A finitarily consistent free-variable positive fragment of Infinitesimal Analysis, Proceedings of the IXth Latin American Symposium on Mathematical Logic Notas de Logica Mathematica 38 (1993), 1-59.

[26] Keita Yokoyama, Complex analysis in subsystems of second order arithmetic, Arch. Math. Logic 46 (2007), no. 1, 15-35. 